\title{
Cancer-associated fibroblasts promote progression and gemcitabine resistance via the SDF-1/SATB-1 pathway in pancreatic cancer
}

Lusheng Wei ${ }^{1,2}$, Huilin Ye ${ }^{1,2}$, Guolin Li ${ }^{1,2}$, Yuanting Lu³, Quanbo Zhou ${ }^{1,2}$, Shangyou Zheng ${ }^{1,2}$, Qing Lin ${ }^{1,2}$, Yimin Liu ${ }^{1,4}$, Zhihua Li ${ }^{1,5}$ and Rufu Chen ${ }^{1,2}$

\begin{abstract}
Cancer-associated fibroblasts (CAFs), a dominant component of the pancreatic tumor microenvironment, are mainly considered as promotors of malignant progression, but the underlying molecular mechanism remains unclear. Here, we show that SDF-1 secreted by CAFs stimulates malignant progression and gemcitabine resistance in pancreatic cancer, partially owing to paracrine induction of SATB-1 in pancreatic cancer cells. CAF-secreted SDF-1 upregulated the expression of SATB-1 in pancreatic cancer cells, which contributed to the maintenance of CAF properties, forming a reciprocal feedback loop. SATB-1 was verified to be overexpressed in human pancreatic cancer tissues and cell lines by quantitative real-time PCR, western blot, and immunohistochemical staining, which correlated with tumor progression and clinical prognosis in pancreatic cancer patients. We found that SATB-1 knockdown inhibited proliferation, migration, and invasion in SW1990 and PANC-1 cells in vitro, whereas overexpression of SATB-1 in Capan-2 and BxPC-3 cells had the opposite effect. Immunofluorescence staining showed that conditioned medium from SW1990 cells expressing SATB-1 maintained the local supportive function of CAFs. Furthermore, downregulation of SATB-1 inhibited tumor growth in mouse xenograft models. In addition, we found that overexpression of SATB-1 in pancreatic cancer cells participated in the process of gemcitabine resistance. Finally, we investigated the clinical correlations between SDF-1 and SATB-1 in human pancreatic cancer specimens. In summary, these findings demonstrated that the SDF-1/ CXCR4/SATB-1 axis may be a potential new target of clinical interventions for pancreatic cancer patients.
\end{abstract}

\section{Introduction}

Pancreatic ductal adenocarcinoma (PDAC) is one of the most lethal and aggressive solid malignancies, with a dismal 5-year survival rate of $<7 \%{ }^{1}$. In America, PDAC is

\footnotetext{
Correspondence: Zhihua Li (zhihuali_sysu@163.com) or Rufu Chen (chenrufu63@163.com)

'Guangdong Provincial Key Laboratory of Malignant Tumor Epigenetics and Gene Regulation, Sun Yat-sen Memorial Hospital, Sun Yat-sen University, Guangzhou, Guangdong Province, China

${ }^{2}$ Department of Pancreatobiliary Surgery, Sun Yat-sen Memorial Hospital, Sun Yat-sen University, Guangzhou, Guangdong Province, China

Full list of author information is available at the end of the article.

These authors contributed equally: Lusheng Wei, Huilin Ye, Guolin Li

Edited by S. Tait
}

the fourth leading cause of cancer-related deaths and is expected to become the second leading cause by $2030^{2}$. The absence of early symptoms and aggressive biological characteristics of tumor are among the reasons for late detection, which makes PDAC act as a silent killer with only $15-20 \%$ of cases diagnosed in the early resectable stages $^{3}$. Poor response to available chemotherapy is another main cause of dismal prognosis. In most patients (74\%), receiving gemcitabine tumor recurrence is eventually observed, with only 13.4 months of disease-free survival $^{4}$. Better understanding of the complex biological

\section{(c) The Author(s) 2018}

(c) (i) Open Access This article is licensed under a Creative Commons Attribution 4.0 International License, which permits use, sharing, adaptation, distribution and reproduction in any medium or format, as long as you give appropriate credit to the original author(s) and the source, provide a link to the Creative Commons license, and indicate if changes were made. The images or other third party material in this article are included in the article's Creative Commons license, unless indicated otherwise in a credit line to the material. If material is not included in the article's Creative Commons license and your intended use is not permitted by statutory regulation or exceeds the permitted use, you will need to obtain permission directly from the copyright holder. To view a copy of this license, visit http://creativecommons.org/licenses/by/4.0/. 
behavior and intricate cellular communication is the prerequisite to developing effective therapeutic strategies.

PDAC is characterized as an abundant desmoplastic tissue that accounts for up to $80 \%$ of total tumor mass ${ }^{5}$. This hallmark feature forms the intra-tumoral microenvironment, which consists of the cancer-associated fibroblasts (CAFs), immune cells, capillaries, basement membrane and extracellular matrix (ECM) surrounding the cancer cells ${ }^{6,7}$. CAFs are the most abundant stromal cell type in pancreatic tumor and are characterized by the expression of activation markers, such as $\alpha$-smooth muscle actin ( $\alpha$-SMA), fibroblast activation protein (FAP), and fibroblast-specific protein 1 (FSP1 $)^{8}$. Activated CAFs in PDAC are variously reported to stem from the pancreatic stellate cells, quiescent resident fibroblasts and mesenchymal stem cells. Indeed, CAFs are also derived from epigenetic transitions from endothelial or cancer cells through endothelial-mesenchymal transition or epithelia-mesenchymal transition (EMT) ${ }^{9,10}$. During the progression of CAF activation, the described pathways involve sonic hedgehog, interleukins 6 and 10, transforming growth factor- $\beta 1$, platelet-derived growth factor (PDGF), basis fibroblast growth factor (bFGF), and other genes $^{7,8}$. CAFs strongly express collagen (type I and III), fibronectin, and hyaluronan, which are the main components of ECM. Increasing evidence indicates that CAFs play an important role in the tumorigenesis, progression, metastasis, and drug resistance ${ }^{11,12}$. However, the biological effects of CAFs on pancreatic cancer progression and chemoresistance remain largely unknown.

Special AT-rich sequence-binding protein 1 (SATB-1) is a nuclear matrix attachment region-binding protein, linking specific DNA elements to its unique cage-like network $^{13}$. SATB-1 can tether genomic loci to the nuclear matrix to form high-order chromatin structure through binding to the AT-rich DNA sequences of base-unpairing regions $^{14}$. SATB-1 also recruits multiple chromatinmodifying enzymes and transcription factors to regulate global gene expression by modifying histones and remodeling nucleosomes ${ }^{13}$. SATB-1 plays a crucial role in the embryonic stem cells and T-cells ${ }^{15,16}$. Han $\mathrm{H}$ et al. ${ }^{17}$ were the first to reveal that SATB-1 promoted breast tumor growth and metastasis. Increasing evidence indicated that SATB-1 upregulation was also closely associated with poor prognosis in other malignancies, such as prostate, ovarian, and gastric cancers, as well as in hepatocellular and renal cell carcinomas ${ }^{18-25}$. Elevated expression of SATB-1 was also associated with poor prognosis in pancreatic cancer ${ }^{26,27}$. However, the specific roles of SATB-1 in CAFs promoted pancreatic cancer progression are poorly elucidated.

In this study, we show that SDF-1, a characteristic C-C chemokine released by tumor-associated fibroblasts, can prominently upregulate the expression of SATB-1 and subsequently contribute to malignant progression and gemcitabine resistance of pancreatic cancer cells. In addition, we have also found that overexpression of SATB-1 in pancreatic cancer cells in turn plays a vital role in maintaining the local supportive function of CAFs, indicating the formation of a SATB-1-centered positive feedback loop in pancreatic cancer. Finally, we examined the clinical correlation of SATB-1 and SDF-1 in human pancreatic cancer specimens. Taken together, our present work provides solid evidence for reciprocal interactions between CAFs and pancreatic cancer cells, shedding new light on the utilization of the SDF-1/CXCR4/SATB-1 axis as a potential therapeutic target for the treatment of pancreatic cancer.

\section{Results}

\section{Characterization of primary NAFs and CAFs}

The human pancreatic normal tissue-associated fibroblasts (NAFs) and CAFs were isolated from fresh pancreatic cancer tissue and adjacent non-neoplastic pancreatic tissue, further confirmed with postoperative cancer tissue by immunohistochemistry staining of $\alpha$ SMA (Fig. 1a). The primary fibroblasts initially showed biand/or multipolar morphology (Fig. 1b). As showed in Fig. 1c, the results of cell immunofluorescence staining indicated that CAFs highly expressed $\alpha$-SMA and FAP, which were not or weakly expressed in NAFs. NAFs and CAFs both expressed vimentin but not cytokeratin 19 (Supplementary Figure 1). To test the purity of NAFs and CAFs, fibroblast biomarkers were examined by quantitative real-time PCR (qRT-PCR), western blot, and immunofluorescence staining. Compared with NAFs, CAFs had high mRNA expression levels of FAP, FSP1, and $\alpha$-SMA (Fig. 1d). Comparing NAFs and CAFs derived from four PDAC patients we detected the protein expression levels of $\alpha$-SMA and FAP to further verify their utility as biomarkers (Fig. 1e). Moreover, we found out that CAFs would be in a worse growth status if CAFs were digested and passed for too many generations. Loss of CAFs markers was also found in CAFs derived from gastric carcinoma $^{28}$. Lack of tumor-secreted factors induction may cause the downregulation of CAFs markers. Then, we performed qRT-PCR and western blot analyses to compare the markers' expression of CAFs at different passages. The results showed that the twelfth-passage CAFs produced lower protein expression of $\alpha$-SMA and FAP compared with the fourth-passage CAFs (Fig. 1f, g). Altogether, these data indicated that we successfully isolated high-purity NAFs and CAFs from PDAC specimens.

\section{SATB-1 was overexpressed in pancreatic cancer cell lines and tissue samples and upregulated by CAFs}

As CAFs demonstrated potent protumor properties in pancreatic cancer, we aimed to characterize the 


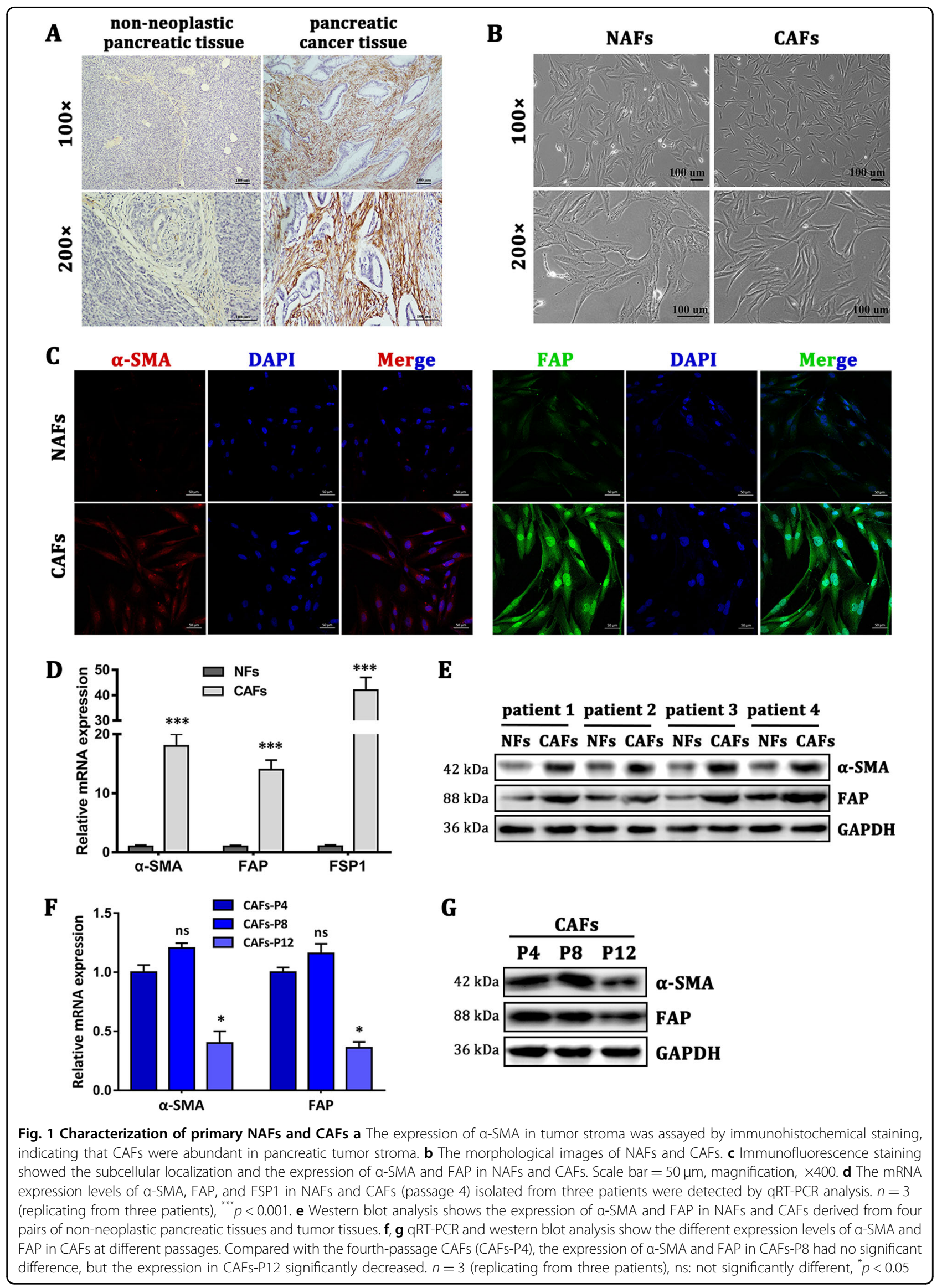


downstream molecular events responsible for CAFmediated malignant progression of pancreatic cancer using the Human $12 \times 135 \mathrm{~K}$ Gene Expression Array manufactured by Roche NimbleGen. Using the transwell co-culture system, we co-cultured PANC-1 pancreatic cancer cells and NAFs or CAFs for 4 days, and subsequently investigated the differential expression profiles of mRNA in PANC-1 cells between the PANC-1 co-cultured with NAFs group (PCN group) and the PANC-1 co-cultured with CAFs group (PCC group). A total of 45,033 mRNA targets were detected by microarray probes in two groups of samples. Overall, 170 mRNAs were evaluated to be differentially modulated by fold change $>2.0$, with the $p$ value $<0.05$ and false discovery rate $<0.05$ (PCC group vs. PCN group). Among them, 131 mRNAs were upregulated, whereas 39 mRNAs were downregulated. In the present study, the top 10 upregulated mRNAs are listed by fold change, among which the cell adhesion molecule SATB-1 was the fourth upregulated gene with $\sim 4.8$-fold change (Table 1). Aberrant expression of SATB-1 has been reported in cells of various cancer types, including prostate cancer, ovarian cancer, gastric cancer, hepatocellular carcinoma, renal cell carcinoma, rectal cancer, bladder cancer, laryngeal squamous cell carcinoma, and cutaneous malignant melanoma ${ }^{29,30}$. However, the specific role of SATB-1 in pancreatic cancer remains elusive. Hence, we chose SATB-1 as a gene of interest and set out to determine whether SATB-1 facilitates malignant progression of pancreatic cancer and participates in the crosstalk between tumor cells and CAFs. qRT-PCR and western blotting showed that SATB-1 was upregulated in PANC-1 and SW1990 pancreatic cancer cells only when co-cultured with CAFs, validating our microarray results (Fig. 2a, b). To investigate SATB-1 mRNA expression levels in PDAC, we performed qRT-PCR analysis on total RNA extracted from 46 pancreatic cancerous tissues and their matched non-neoplastic counterparts. Our current results showed that SATB-1 mRNA was significantly overexpressed in pancreatic cancerous samples in comparison with those of corresponding normal tissues (Fig. 2c). Subsequently, we randomly selected 12 paired PDAC samples to evaluate the SATB-1 protein expression level using western blot analysis. In agreement with the PCR observations, the SATB-1 protein level was significantly upregulated in pancreatic cancerous tissues (Fig. 2d). Moreover, seven PDAC cell lines (PANC-1, SW1990, Capan-2, CFPAC-1, BxPC-3, AsPC-1, and MIAPaCa-2) also showed significantly higher SATB-1 mRNA and protein levels than the pancreatic ductal epithelium cell line HPDE6-C7, with the two highest expression levels observed in PANC-1 and SW1990 cells (Figure E-F). Although Capan-2 and Bxpc-3 expressed lower SATB-1, they would also upregulate the SATB-1 expression in CAFs co-culture system (Supplementary
Figure 2). In summary, these results manifested that CAFs can significantly upregulate the expression of SATB-1 in pancreatic cancer cells and that SATB-1 is markedly increased in both pancreatic cancerous tissues and pancreatic cancer cell lines.

\section{SDF-1 secretion is related to CAF-induced SATB-1 upregulation in pancreatic cancer cells}

In many cocultures with different types of tumor cells, CAFs enhanced tumorigenesis, metastasis, or drug resistance of cancer cells via secretion of many soluble factors ${ }^{9}$. Then, we analyzed the mRNA expression of PGDF-A, VEGF, IGF-1, hepatocyte growth factor (HGF), bFGF, SDF-1, IL-6, CCL18, TNF$\alpha$, and TGF- $\beta 1$ of NAFs and CAFs (Supplementary Figure $3 \mathrm{~A}$ ). We found that TGF- $\beta 1$ and SDF- 1 were two of the highest cytokines secreted by CAFs, similar to previous study ${ }^{31}$. Then, we performed enzymelinked immunosorbent assay (ELISA) analysis to further confirm the secretion of TGF- $\beta 1$ and SDF- 1 in CAFs-CM compared with matched NAFs-CM (Supplementary Figure 3B). CAFs-secreted cytokines may promote the expression of SATB-1 in pancreatic cancer cells. Therefore, we analyzed the mRNA expression of SATB-1 in PANC-1 and SW1990 cells after the induction of human recombinant TGF- $\beta 1$ and SDF-1. We found that recombinant SDF-1 upregulated SATB1 expression 3.28-fold, whereas recombinant TGF- $\beta 1$ produced no change (Fig. 3a, b). Previous studies have reported that CXCR4 is the main cognate receptor of SDF-1, and the SDF-1/CXCR4 biological axis has an important role in many types of solid tumors, including pancreatic, breast, gastric, and lung cancers. Therefore, we examined whether the SDF-1/CXCR4 axis regulates the SATB-1 expression level in pancreatic cancer cells. We analyzed the SATB-1 expression level in SW1990 and PANC-1 cells after an addition of human recombinant SDF-1 or conditioned medium derived from CAFs (CM-CAFs), or after indirect coculturing with CAFs. The mRNA expression level of SATB-1 was upregulated by SDF-1, CM-CAFs, and CAFs (Fig. 3c). The western blot data showed a similar result (Fig. 3d). Moreover, when SDF-1 was neutralized, the level of SATB-1 protein in SW1990 and PANC-1 cells similar to that in SW1990 and PANC-1 cells cultured alone. On the other hand, when silencing CXCR4 with siRNA before treating cells with SDF-1, the effect of SDF-1 on upregulation of the SATB-1 expression level was abrogated, as evidenced by the return of SATB-1 mRNA and protein level to the baseline level (Figure $3 g-h)$. In conclusion, these data demonstrated that CAFs upregulated the expression level of SATB-1 in pancreatic cancer cells through the SDF-1/CXCR4 axis. 
Table 1 Top 10 upregulated mRNAs in PANC-1 cells co-cultured with CAFs

\begin{tabular}{|c|c|c|c|c|}
\hline Fold change & $P$ value & Regulation & Gene Symbol & Description \\
\hline 5.7183622 & 0.000441 & Up & OR9G1 & Olfactory receptor family 9 subfamily G member 1 \\
\hline 5.3407117 & 0.035397 & Up & RASAL3 & RAS protein activator like 3 \\
\hline 4.9443596 & 0.029836 & Up & ARHGAP30 & Rho GTPase activating protein 30 \\
\hline 4.8004442 & 0.014924 & Up & SATB-1 & SATB homeobox 1 \\
\hline 4.3003547 & 9.53E-06 & Up & TJP3 & Tight junction protein 3 \\
\hline 4.1499271 & $1.45 \mathrm{E}-06$ & Up & $\mathrm{SH} 2 \mathrm{D} 3 \mathrm{C}$ & $\mathrm{SH} 2$ domain containing $3 \mathrm{C}$ \\
\hline 3.8759635 & 0.032572 & Up & FNDC3A & Fibronectin type III domain containing 3A \\
\hline 3.7832849 & 0.020696 & Up & DEF6 & DEF6, guanine nucleotide exchange factor \\
\hline 3.6076283 & 0.010234 & Up & C19orf45 & Chromosome 19 open reading frame 45 \\
\hline 3.5313205 & 0.005514 & Up & IP6K3 & Inositol hexakisphosphate kinase 3 \\
\hline
\end{tabular}

SATB-1 mediates the proliferation and G1-S checkpoint of pancreatic cancer cells in vitro

To further investigate the impact of SATB-1 on pancreatic cancer progression, in vitro functional characterizations were performed. First, qRT-PCR assay and western blot analysis indicated that SATB-1 expression was significantly reduced by specific small interfering RNAs (siRNAs) in SW1990 and PANC-1 cells and upregulated after transfection of pcDNA3.1-SATB-1 cDNA vector into Capan-2 and BXPC-3 cells (Fig. 4a, b). Compared with control cells, SATB-1 downregulation significantly inhibited tumor cell proliferation in SW1990 and PANC-1 cells, as shown by the CCK- 8 assay. In contrast, SATB-1 overexpression had an opposite effect (Fig. 4c). In addition, we performed the flow cytometry analysis for the distribution of cell cycle. The results demonstrated that downregulation of SATB-1 led to a substantial accumulation of PDAC cells in G0/G1 phase, accompanied by a substantial decrease in $S$ phase (Fig. $4 d$, e). SATB-1 overexpression, however, had an opposite influence on the cell cycle distribution in Capan-2 and BxPC-3 cells (Fig. 4f, g). Interestingly, SATB-1 downregulation in PDAC cells made no difference in the fraction of apoptotic cells (Supplementary Figure 4). Altogether, SATB-1-induced acceleration of PDAC cell proliferation appeared to be facilitated by variation of the G1-S checkpoint, rather than by cell apoptosis.

SATB-1 promotes PDAC cell migration and invasion in vitro

Enhanced cell migration and invasion abilities underlie the mechanisms of cancer metastasis, resulting in poor prognosis. Our wound-healing scratch assay clarified that SATB-1 knockdown in PANC-1 and SW1990 cells markedly decreased cell motility compared with the si-NC control groups, whereas the coculturing with CAFs promoted the capability of healing. Then, the wound-healing postponed when anti-SDF-1 antibodies neutralized the SDF-1 derived from CAFs in the co-culture system (Fig. 5a). Further, the transwell assay demonstrated that downregulation of SATB-1 prominently weakened the migration and invasion of PANC-1 and SW1990 cells, whereas CAFs strengthened these capabilities of PANC-1 and SW1990 cells (Fig. 5b). In contrast, overexpression of SATB-1 significantly enhanced the migration and invasion of Capan-2 and BxPC-3 cells (Fig. 5c), as indicated by wound-healing and transwell analyses. These observations verified that SATB-1 enhanced PDAC cell migration and invasion via CAF-secreted SDF-1 in vitro.

\section{Overexpression of SATB-1 in pancreatic cancer cells reciprocally sustains the hallmark of CAFs in vitro}

As CAFs exert significant impact on the metastatic hallmark of pancreatic cancer, we wondered whether overexpression of SATB-1 in pancreatic cancer cells could influence the fibroblast phenotype. We cultured CAFs with CM from either control pancreatic cancer cells or SATB-1-silenced pancreatic cancer cells and analyzed the $\alpha$-SMA and FAP expression levels in CAFs. First, qRTPCR assay indicated that the mRNA expression of $\alpha$ SMA, FAP, and SDF-1 was significantly increased in CAFs-P4 and CAFs-P12 induced with CM from SW1990control cells and SATB-1-overexpressed BxPC-3 cells compared with control CAFs (Fig. 6a). However, conditioned medium from SATB-1-silenced SW1990 cells did not increase the expression of hallmark mRNAs in CAFsP4 and CAFs-P12. Moreover, western blot and immunocytochemistry staining further confirmed the above results (Fig. 6b, c). Then, NAFs were also treated with conditioned medium from pancreatic cancer cells with different SATB-1 expression. The result indicated that SATB-1 expression was also involved in the activation of CAFs from NAFs (Supplementary Figure 5). These data 

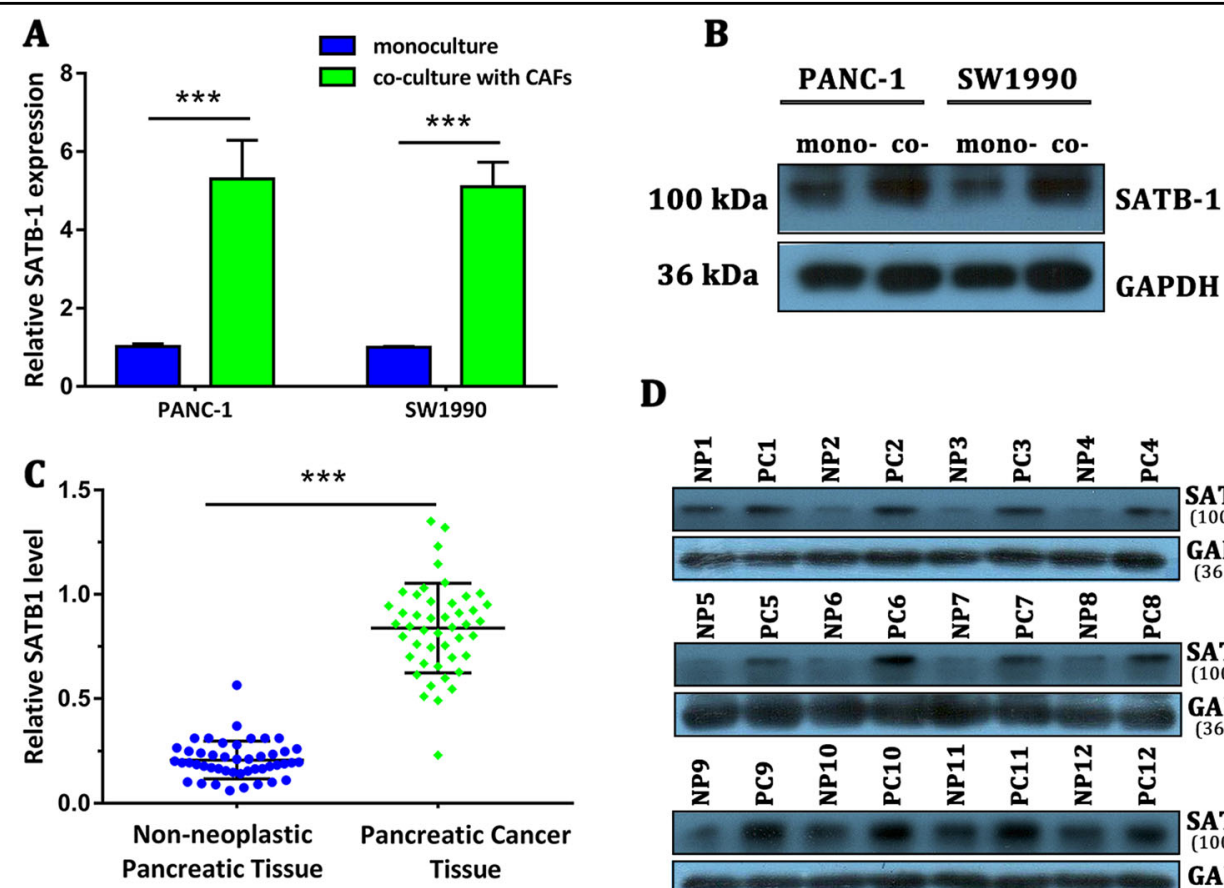

D
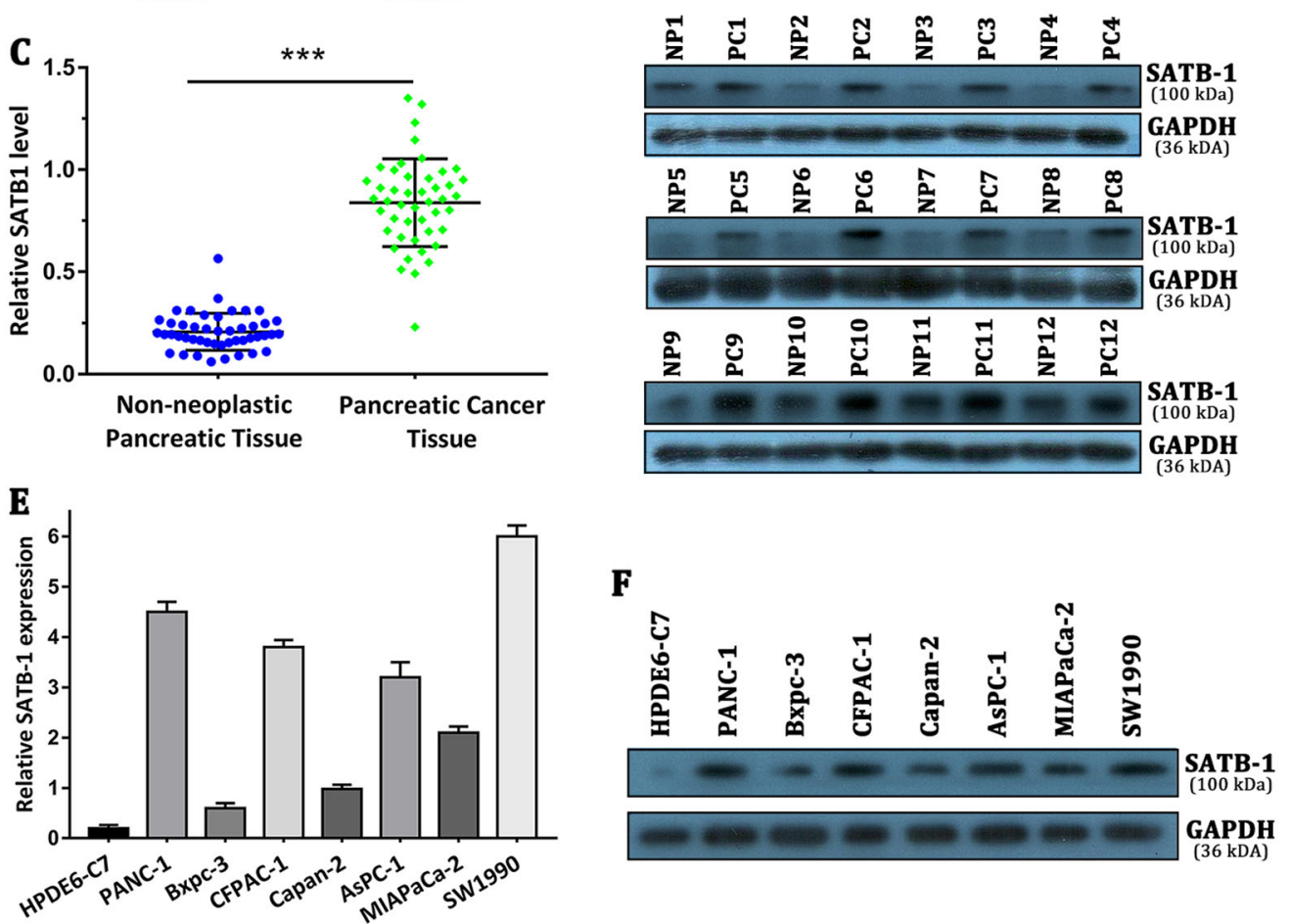

Fig. 2 SATB-1 is overexpressed in pancreatic cancer cell lines and tissue samples and is upregulated by CAFs a, $\mathbf{b}$ The qRT-PCR and western blot analysis show the mRNA and protein levels of SATB-1 in SW1990 and PANC-1 cells cultured with (co-culture) or without CAFs (monoculture). $n=$ $3,{ }^{* * *} p<0.001$. c The qRT-PCR analysis shows the mRNA expression of SATB-1 in 32 pancreatic tumor tissues and matched non-neoplastic pancreatic tissues. Dots represent each patient, and error bars indicate standard deviation (SD). $n=3,{ }^{* * *} p<0.001$. d Western blot analysis shows the protein levels of SATB-1 in 12 pancreatic cancer tissues (PC) and matched non-neoplastic pancreatic tissues (NP). e, $\mathbf{f}$ The qRT-PCR and western blot analyses show the mRNA and protein levels of SATB-1 in various pancreatic cancer cell lines. $n=3$

suggest that overexpression of SATB-1 in pancreatic cancer cells reciprocally sustains CAF-like features and contributes to the malignant characteristics of the fibroinflammatory tumor microenvironment.

\section{SATB-1 mediates gemcitabine resistance in PDAC cells in vitro}

Resistance to anticancer drugs critically limits the effectiveness of chemotherapy. Gemcitabine is the standard chemotherapy drug for pancreatic cancer ${ }^{32}$. To investigate whether overexpression of SATB-1 in PDAC cells correlates with an active role in gemcitabine resistance, we measured the half-maximal inhibitory concentration (IC50) of gemcitabine in SW1990 and PANC-1 cells under various conditions (Fig. 7a, b). Downregulation of SATB-1 decreased the IC50 values in pancreatic cancer cells, thus indicating that SATB-1 played an active role in promoting gemcitabine resistance in pancreatic cancer cells. Moreover, an addition of both exogenous recombinant SDF-1 and conditioned medium derived from CAFs (CAFs-CM) increased the IC50 values, whereas the promoting effect of CAFs-CM was not observed in the presence of a neutralizing antibody against SDF-1. The IC50 values were calculated as shown in Fig. 7c. We further observed the morphological changes of SW1990 and PANC-1 incubated with gemcitabine at various concentrations, and the surviving cells came shrinking with elongated pseudopodiums (Fig. 7d). 
A

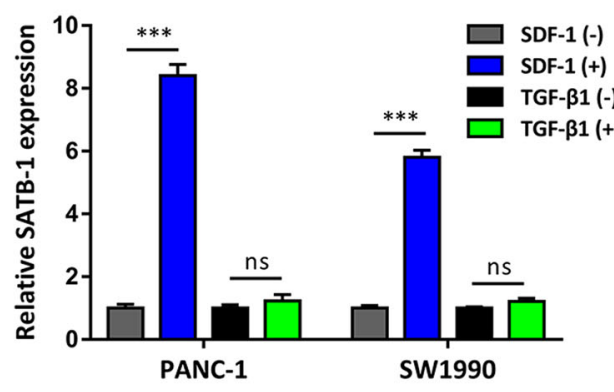

C

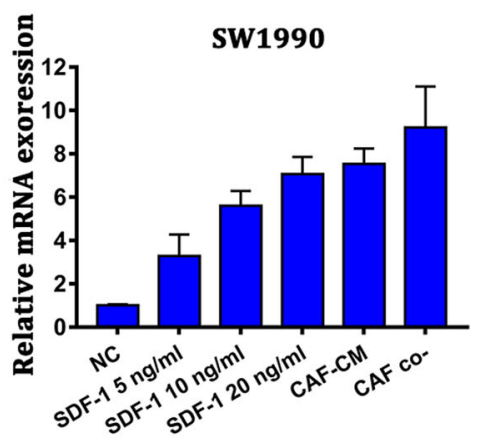

E

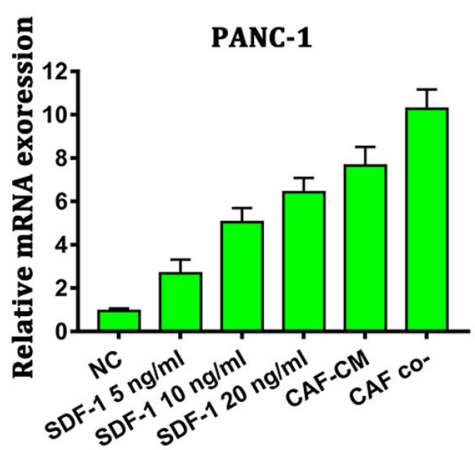

G

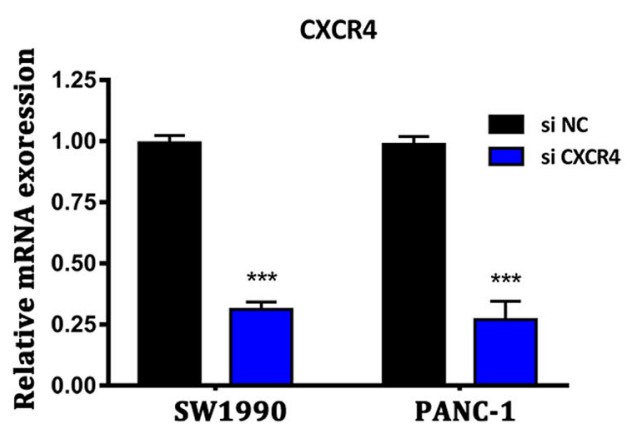

B
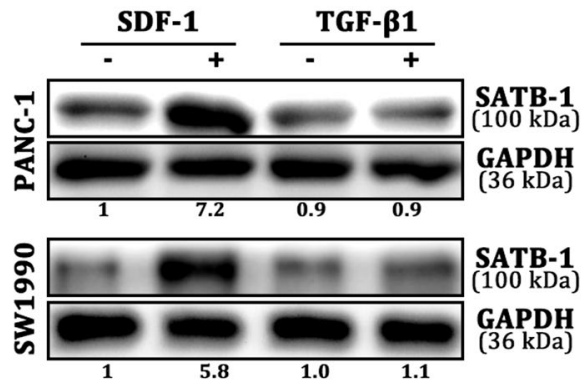

D

SW1990

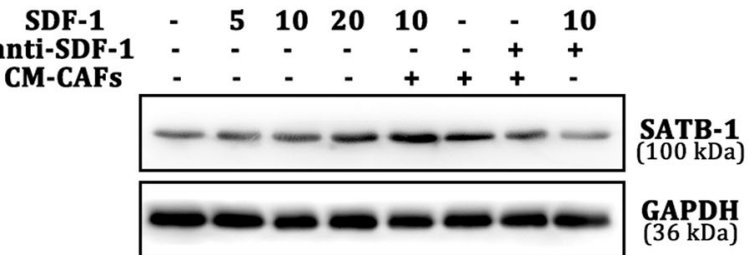

F

PANC-1

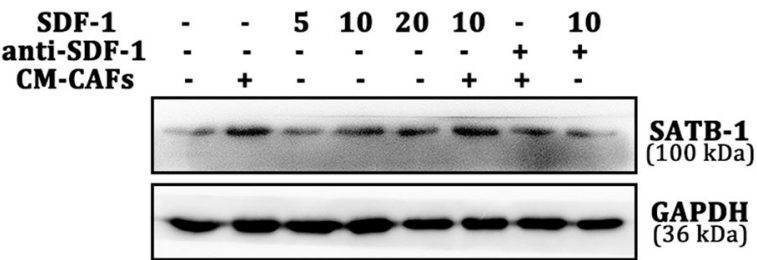

H

$\frac{+ \text { SDF-1 }}{\operatorname{sinC} \text { si-CXCR4 }} \frac{+ \text { CAFs Co- }}{\text { SiNC si-CXCR4 }}$
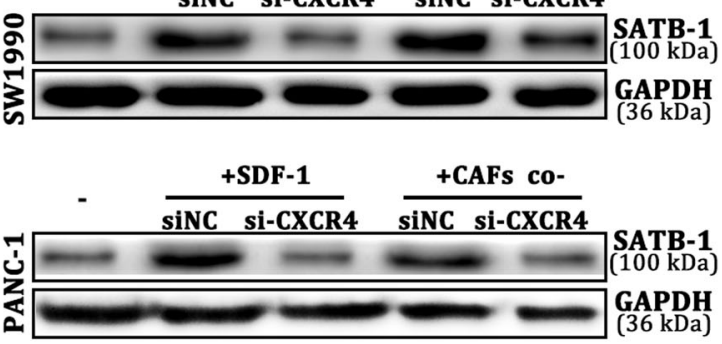

Fig. 3 SDF-1 secretion is related to CAF-induced SATB-1 upregulation in pancreatic cancer cells $\mathbf{a}$, $\mathbf{b}$ The qRT-PCR and western blot analysis show the mRNA expression and protein levels of SATB-1 in SW1990 and PANC-1 cells after the induction of SDF- 1 and TGF- $\beta 1 . n=3, n s:$ not significantly different, ${ }^{* * *} p<0.001$. c, d The qRT-PCR and western blot analyses show the mRNA and protein levels of SATB-1 in SW1990 cells with the addition of various concentrations of SDF-1 and CM-CAFs. e, $\mathbf{f}$ The qRT-PCR and western blot analyses show the mRNA and protein levels of SATB-1 in PANC-1 cells with the addition of various concentration of SDF-1, CM-CAFs, or anti-SDF-1 (4 $\mu \mathrm{g} / \mathrm{ml})$. $\mathbf{g}$ The qRT-PCR analysis indicates that the expression of CXCR4 in SW1990 and PANC-1 cells was effectively downregulated. $n=3,{ }^{* * *} p<0.001$. $\mathbf{h}$ The western blot analysis shows the protein levels of SATB-1 in SW1990 and PANC-1 cells with CXCR4 silenced. SW1990 and PANC-1 cells were incubated with SDF-1 for 4 days or co-cultured with CAFs for 4 days 
A

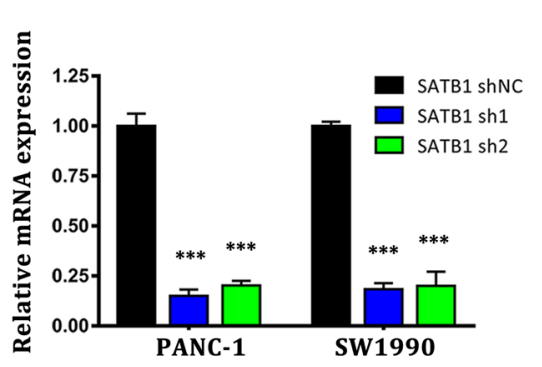

C

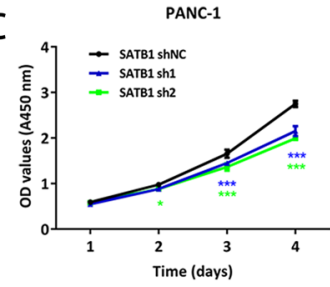

D
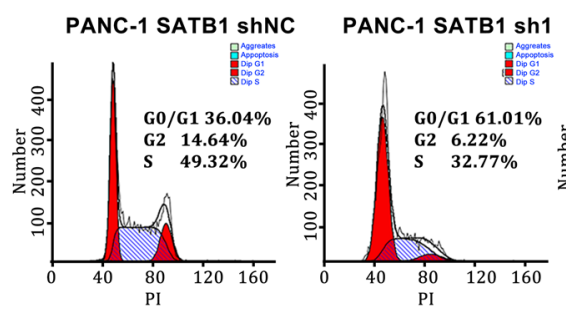

SW1990
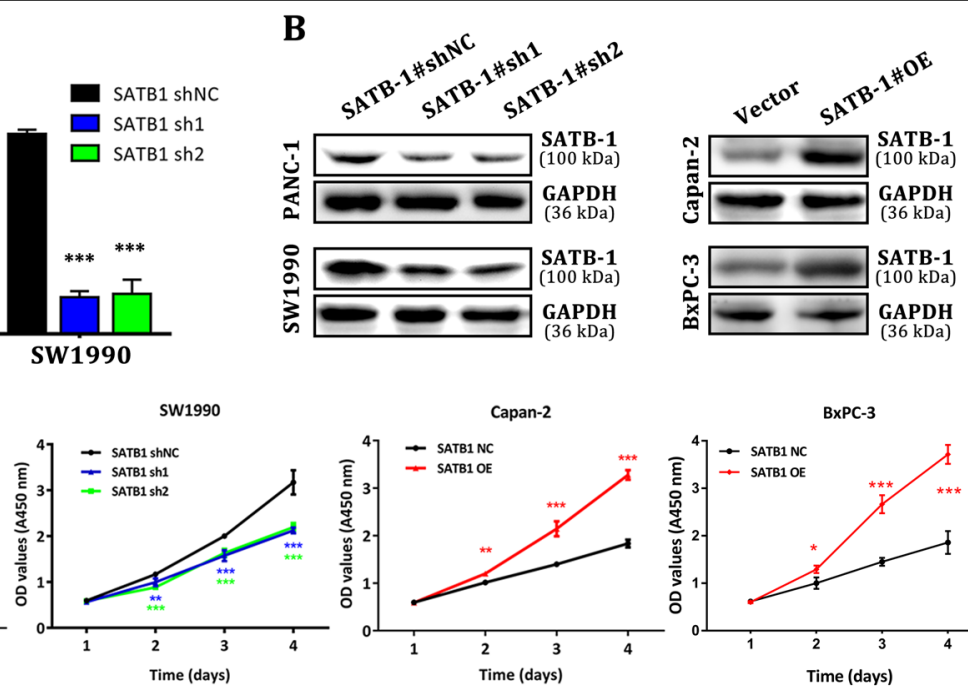

E
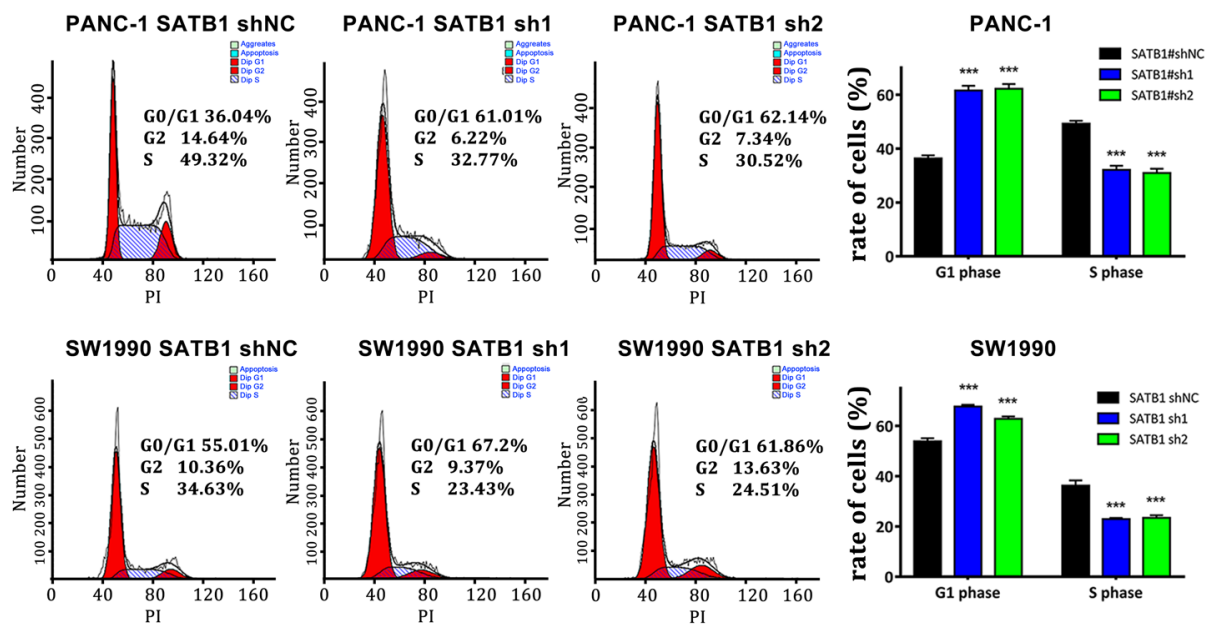

F
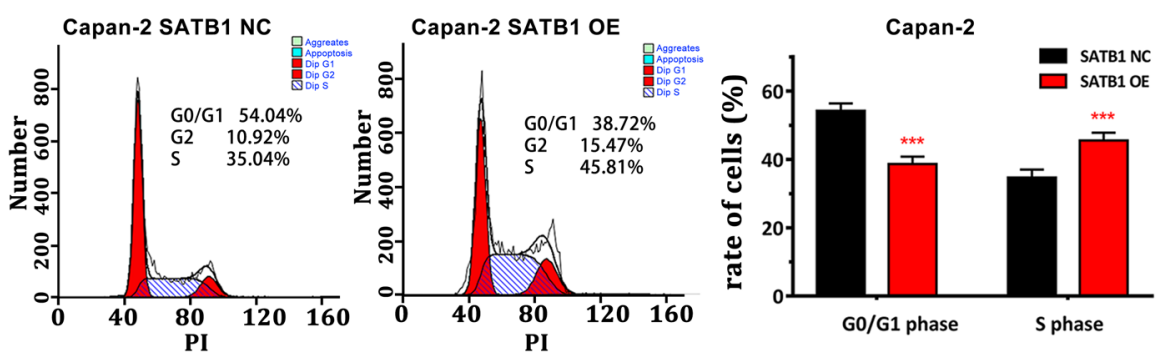

G
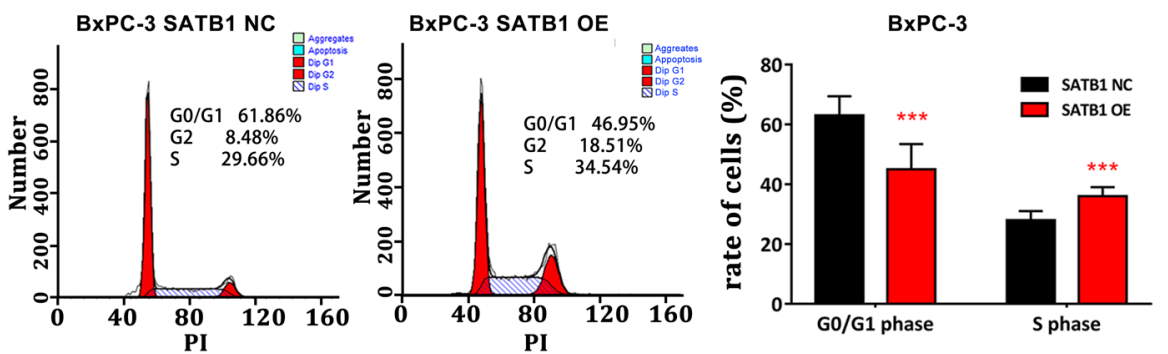

Fig. 4 SATB-1 mediates the proliferation and G1-S checkpoint of pancreatic cancer cells in vitro a, b The qRT-PCR and western blot analyses show that the expression levels of SATB-1 in PANC-1 and SW1990 cells with specific sh-RNA were downregulated. SATB-1 was overexpressed at the protein level in Capan-2 and BXPC-3 cells transfected with pCDNA3.1-SATB-1. $n=3,{ }^{* * *} p<0.001$. c CCK-8 analysis shows the growth curve of PANC-1 and SW1990 cells with SATB-1 stably silenced and the growth curve of Capan-2 and BXPC-3 cells overexpressing SATB-1. $n=3,{ }^{*} p<0.05,{ }^{* *} p<0.01$, ${ }^{* * *} p<0.001$. d, e FACS analysis shows the impact of SATB-1 on cell cycle distribution of PANC-1 and SW1990. $n=3,{ }^{* * *} p<0.001$. f-g FACS analysis shows the impact of SATB-1 on cell cycle distribution of Capan-2 and BXPC-3 cells. $n=3,{ }^{* * *} p<0.001$ 
$\mathbf{A}$
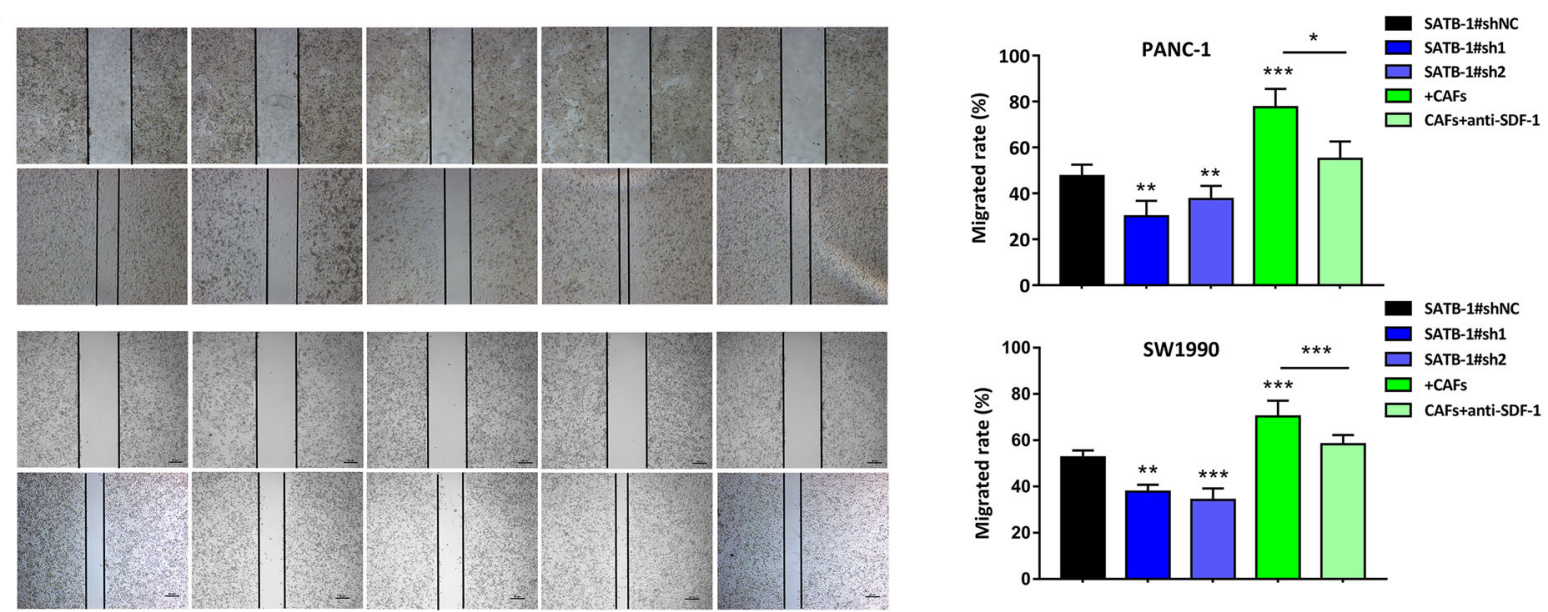

B
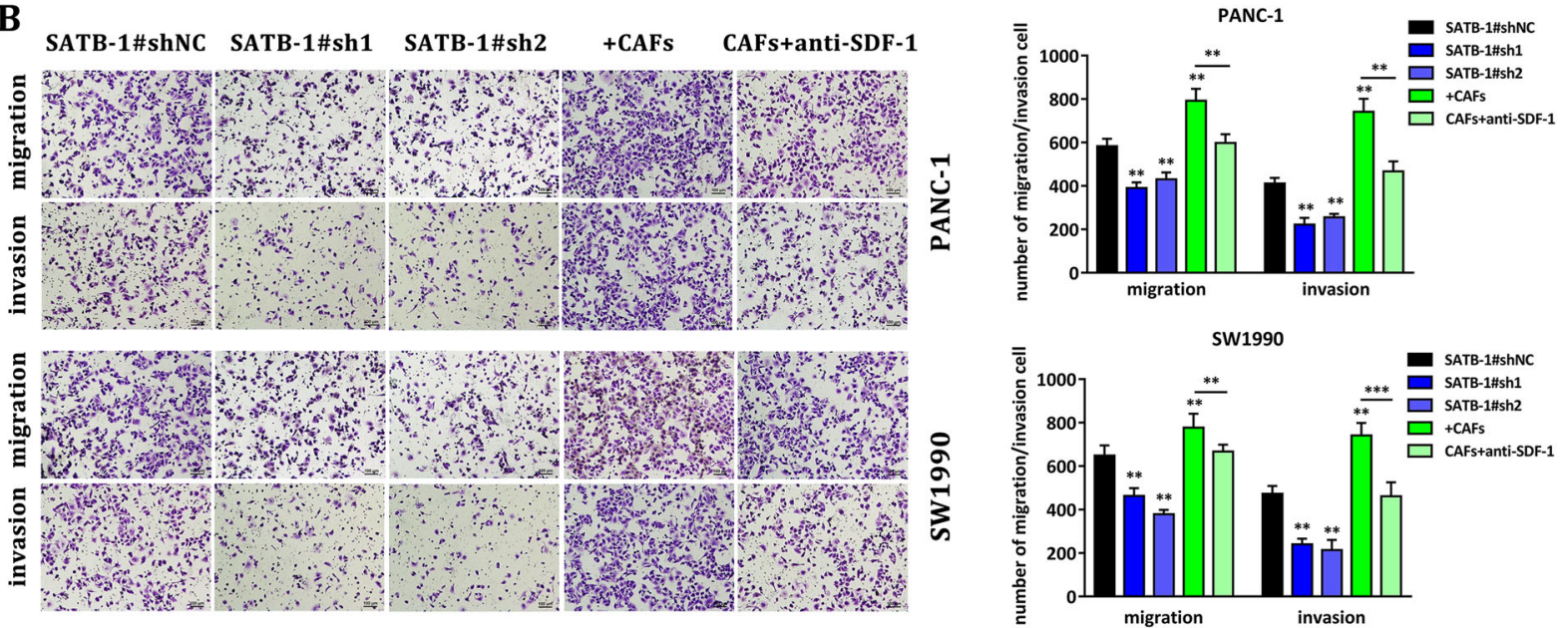

C
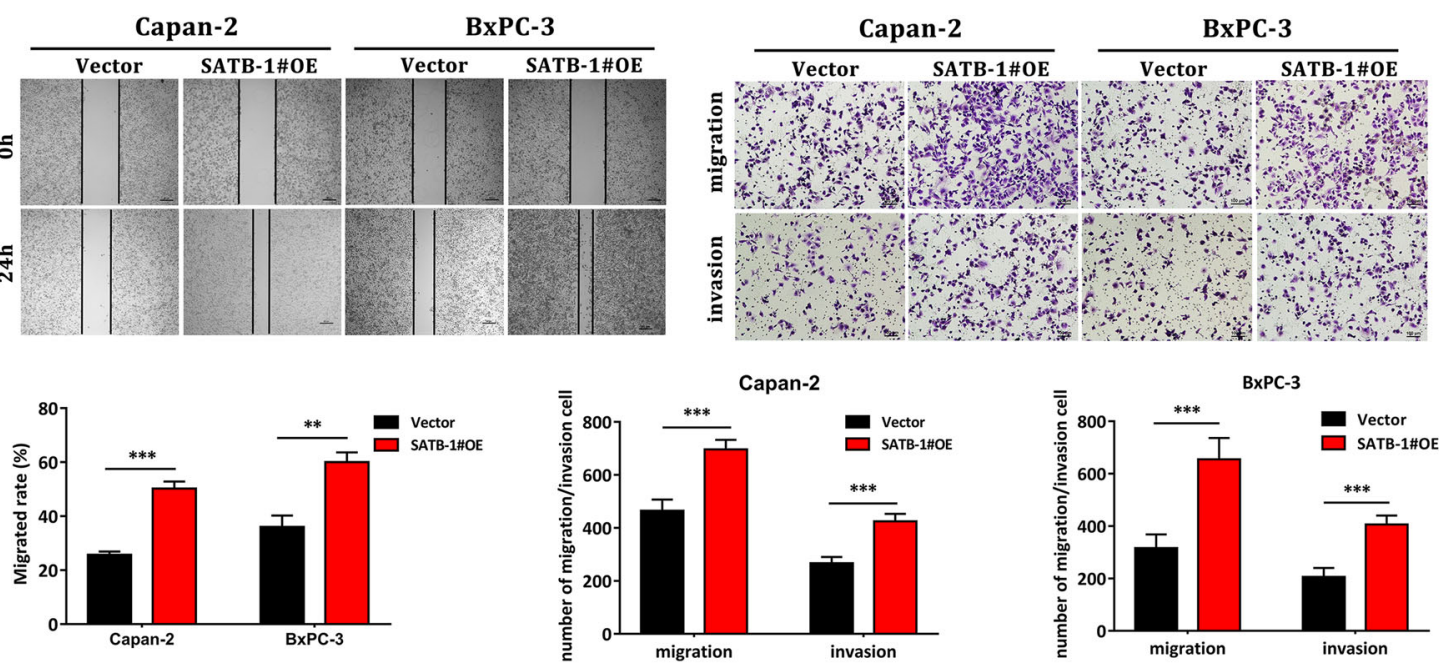

Fig. 5 SATB-1 enhanced the migration and invasion abilities of pancreatic cancer cells a Wound-healing assay shows the abilities of SW1990 and PANC-1 cells with SATB-1 silenced or co-cultured. $\mathbf{b}$ The transwell assay shows the fractions of migrated and invaded SW1990 and PANC-1 cells with SATB-1 silenced or co-cultured. Knockdown of SATB-1 inhibited the migration and invasion abilities of PANC-1 and SW1990 cells. Coculturing with CAFs enhanced the migration and invasion abilities of PANC-1 and SW1990 cells, but neutralization with anti-SDF-1 antibody reduced these abilities. Compared with control pancreatic cancer cells, $n=3,{ }^{*} p<0.05,{ }^{* *} p<0.01,{ }^{* * *} p<0.001$. c Wound-healing and transwell assays show the upregulated migration and invasion abilities of Capan-2 and BXPC-3 cells transfected with PCDNA3.1-SATB-1. Compared with control pancreatic cancer cells, $n=3,{ }^{* *} p<0.01,{ }^{* * *} p<0.001$ 


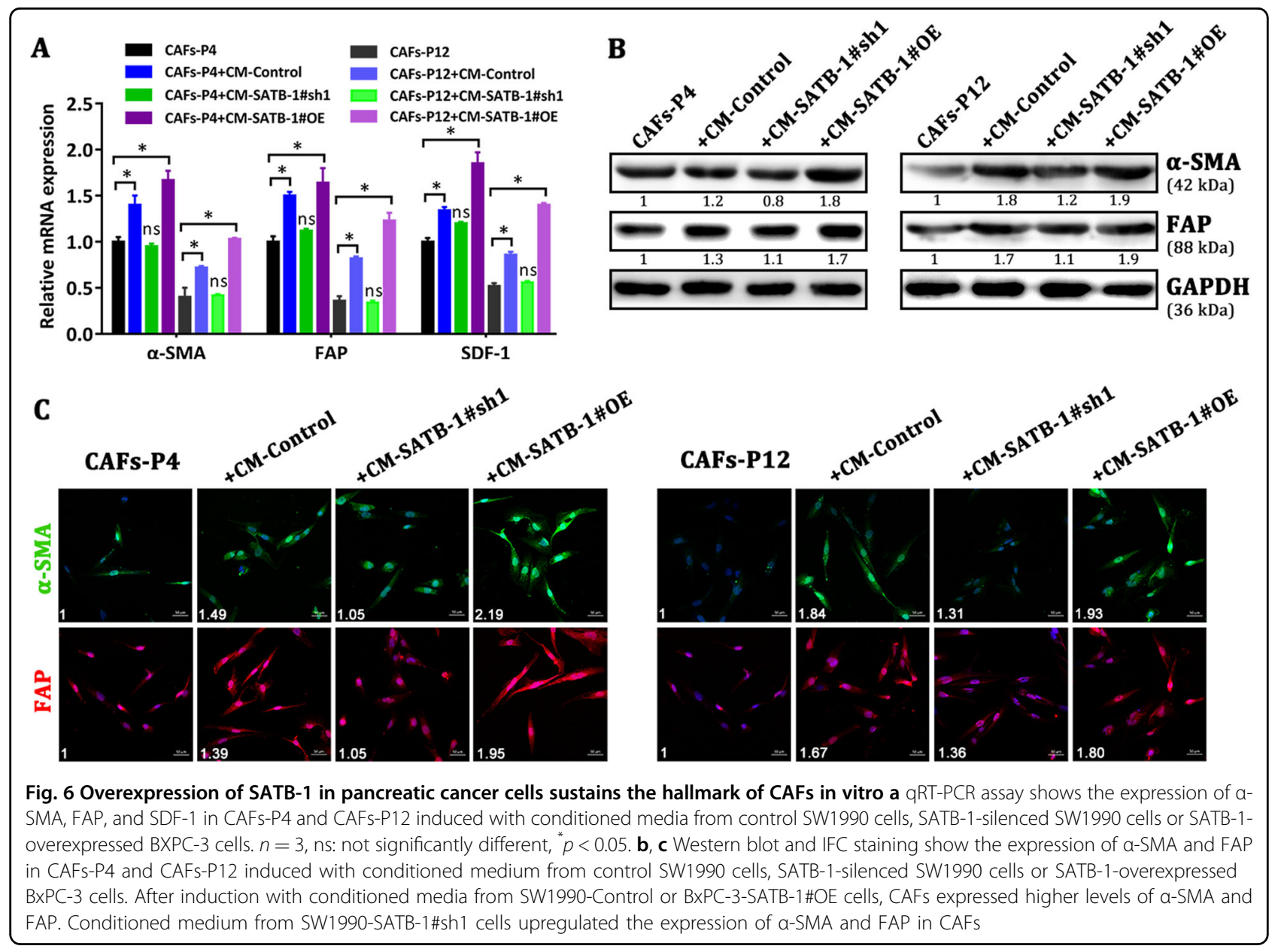

Together with the data presented above, these results suggested that CAFs induced the gemcitabine resistance in pancreatic cancer cells by upregulation of SATB-1 via CAF-secreted SDF-1.

SATB-1 is important for pancreatic tumor growth in vivo

To investigate whether SATB-1 played a role in tumor formation in vivo, we performed a subcutaneous injection of SW1990 cells with stable SATB-1 knockdown (SATB$1 \#$ sh1) or mock-transfected cells (sh-NC) into the bilateral hind legs of athymic nude mice. A month later, all mice developed xenograft tumors at the injection site (Fig. 8a, b). By qRT-PCR analysis and western blot assay, we confirmed the SATB-1 knockdown in the xenotransplanted tumors in the sh-SATB-1 group (Fig. 8e, f). Immunostaining showed that tumors in the sh-SATB-1 group inhibited positive expression of Ki-67 compared with the sh-NC group (Fig. 8g). Consistent with the in vitro results, xenograft tumors grown in SATB-1silenced SW1990 cells had a smaller mean size and weight than xenograft tumors grown in mock cells (Fig. 8c, d). The growth of implanted tumor with SW1990-SATB1\#sh2 was inhibited (Supplementary Figure 6). Taken together, these findings indicated that SATB-1 played a vital role in PDAC proliferation capacity in mouse xenograft models.

\section{SDF-1 and SATB-1 overexpression correlates with} clinicopathological characteristics and poor prognosis of PDAC patients

To investigate the clinical relevance of the SDF-1/ SATB-1 axis in pancreatic cancer, we analyzed the expression levels of SDF-1 and SATB-1 protein in 243 paraffin-embedded human PDAC samples by immunohistochemistry. As described in the Methods, the expression levels of SDF-1 and SATB-1 were separately evaluated according to the intensity and percentage and finally stratified into two groups (low and high expression groups; score range of 0-12). Figure 9a shows representative images of these two groups. The relationship between the intensity of SDF-1 or SATB-1 staining and clinicopathological features was also analyzed. Statistical analysis confirmed that SDF-1 overexpression only correlated with the lymph node metastasis, whereas SATB-1 overexpression correlated with differentiation, $\mathrm{T}$ stage, TNM stage, and lymph node metastasis (Table 2). 

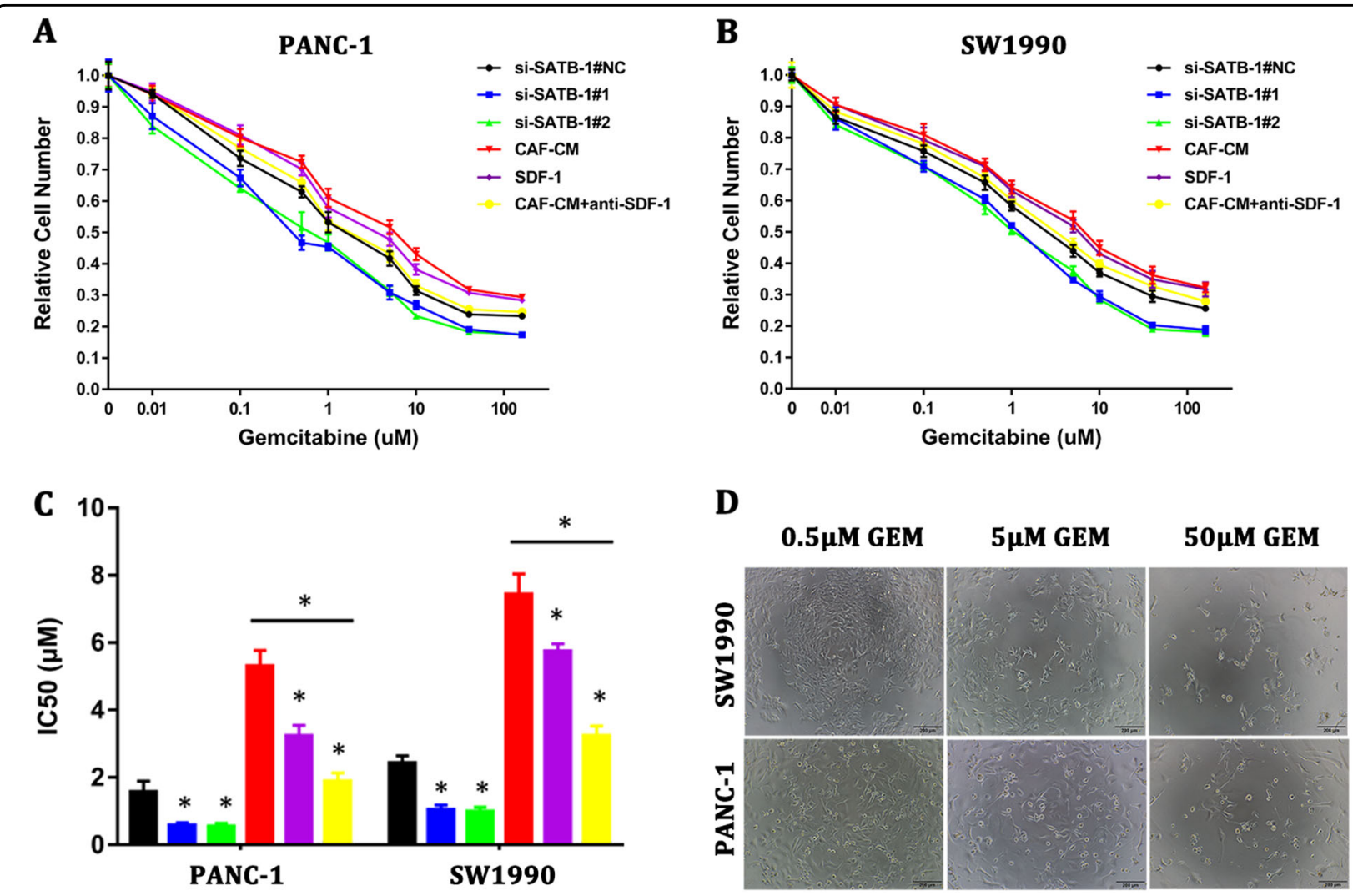

Fig. 7 SATB-1 mediated gemcitabine resistance in pancreatic cancer cells in vitro $\mathbf{a}, \mathbf{b}$ CCK- 8 assay showed the survival rate of PANC-1 and SW1990 cells induced with various concentrations of gemcitabine in different conditions. $n=3$. c The chart shows the IC50 values for PANC-1 and SW1990 cells induced with various concentrations of gemcitabine in different conditions. $n=3,{ }^{*} p<0.05$. $\mathbf{d}$ The images show the morphological changes of PANC-1 and SW1990 cells induced with various concentrations of gemcitabine

Furthermore, the Kaplan-Meier survival plots displayed that higher SDF-1 or SATB-1 staining intensity correlated with poorer prognosis in PDAC patients (log-rank test, $p<0.001$, Fig. 9b). Moreover, the univariate analysis showed that tumor differentiation, T stage, TNM stage, lymph node metastasis, SATB-1 expression, and SDF-1 expression were significantly associated with an increased risk of cancer-related death. The multivariate analysis indicated that $\mathrm{T}$ stage, TNM stage, lymph node metastasis, SATB-1 expression and SDF-1 expression were independent prognostic factors (Table 3). To investigate the relationship between SDF-1 expression and SATB-1 expression, Chi-Square test was used to analyze and the result showed that those patients with higher SDF-1 expression tended to express higher SATB-1 $(p<0.05)$ (Table 4).

\section{Discussion}

PDAC is one of malignant tumors with aggressive progression and high mortality rate. As a scirrhous carcinoma, abundant stromal content is one of PDAC hallmark features. CAFs play an important role in the tumor microenvironment, which has been proven to contain an autocrine-paracrine communication loop that impels tumor growth and metastasis ${ }^{33-35}$. In this study, we demonstrated that SDF-1, also known as C-X-C motif chemokine 12 (CXCL12), secreted by CAFs upregulated SATB-1 expression in pancreatic cancer cells via the SDF1/CXCR4 axis. Then, we found that SDF-1-induced SATB-1 upregulation inhibits proliferation, migration, and invasion of pancreatic cancer cells, and conditioned medium from SW1990 and PANC-1 cells expressing SATB-1 maintains the properties of older CAFs, forming a SATB-1-centered positive feedback loop. Furthermore, we found that the expression of SATB-1 participates in the process of gemcitabine resistance. Finally, we tested the clinical correlations in human pancreatic cancer specimens. Our results revealed that the SDF-1/CXCR4/ SATB-1 axis is vital to acceleration of the malignant progression and the gemcitabine resistance of pancreatic cancer cells and is associated with poor prognosis in PDAC patients.

Generally, CAFs are considered as positive mediators in the tumor microenvironment by secreting cytokines, chemokines, and proangiogenic factors. These mainly include, but not limited to, SDF-1, TGF- $\beta 1$, 
A

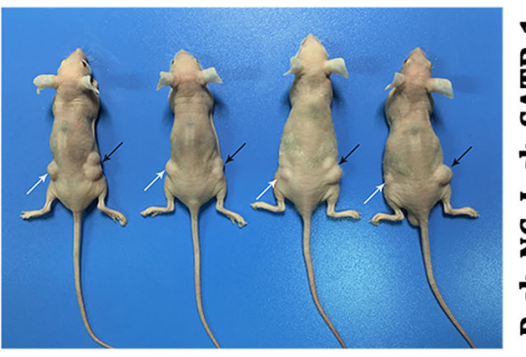

C

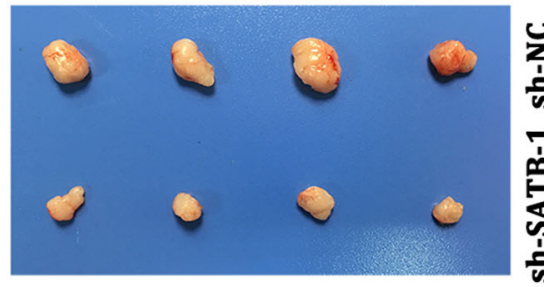

E

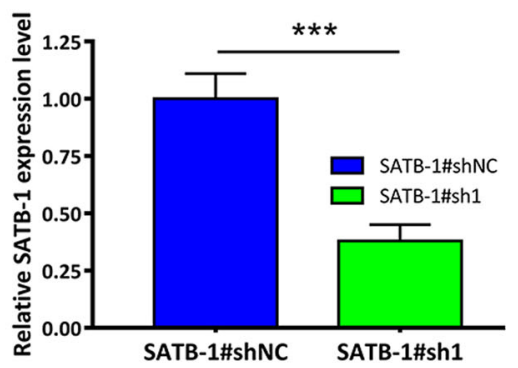

B

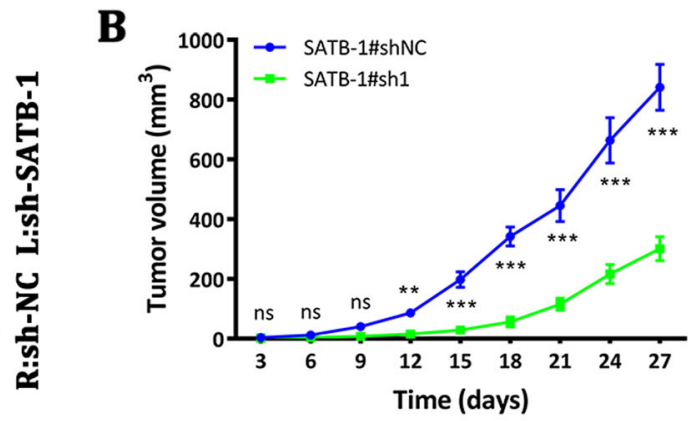

D

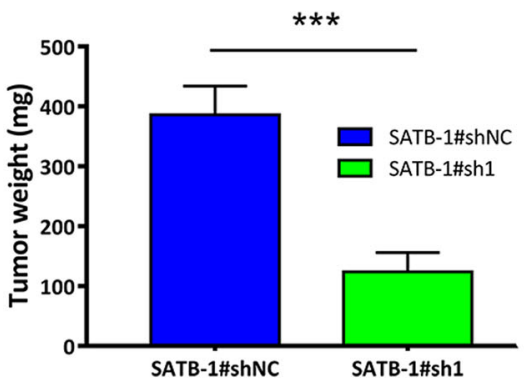

F

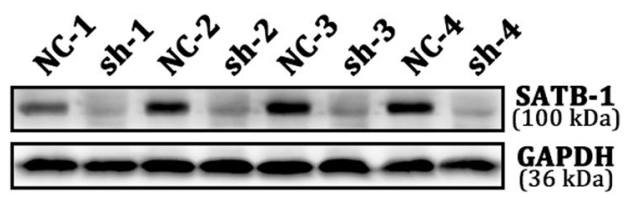

G

sh-NC

sh-SATB-1
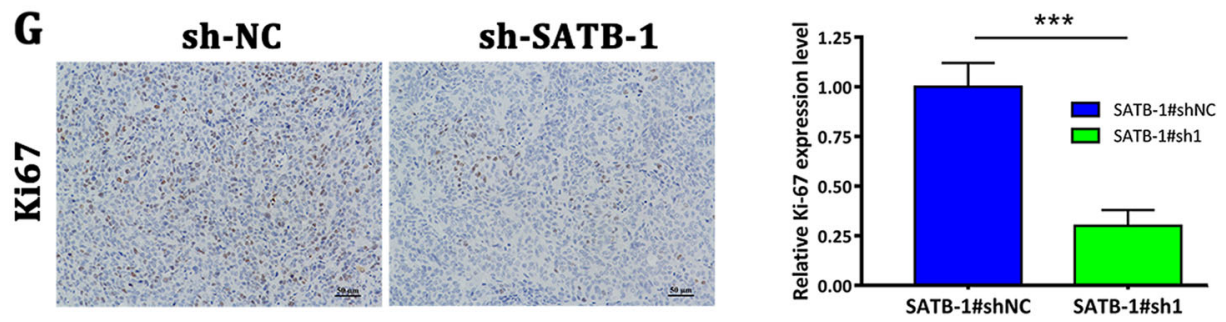

Fig. 8 Knockdown of SATB-1 inhibits tumor growth in mouse xenograft models a SW1990 cells with a stable SATB-1 knockdown or mock cells were subcutaneously injected into the bilateral hind legs of nude mice. At 27 days after injection, SW1990 cells transfected with sh-SATB-1 (white arrow) and mock cells (black arrow) developed primary tumors. $\mathbf{b}$ Tumor growth curve. The points and bars represent means \pm SD. $n=4$, ns: not significantly different. ${ }^{* *} p<0.01,{ }^{* * *} p<0.001$. c The images of harvested tumors. $\mathbf{d}$ Tumor weights are shown as means \pm SD. $n=4,{ }^{* * *} p<0.001$. e, $\mathbf{f}$ The qRT-PCR and western blot assay analyzed the mRNA and protein expression levels of SATB-1 in tumor tissues from sh-SATB-1 SW1990 cells compared with sh-NC SW1990 cells. g IHC staining showed the reduced expression of Ki-67, a proliferation marker, in tumor samples from sh-SATB-1 SW1990 cells. $n=4,{ }^{* * *} p<0.001$

heat shock factor 1, HGF, IL-6, tumor necrosis factor (TNF), and $\mathrm{C}-\mathrm{C}$ motif chemokine ligand $2^{7}$. SDF- 1 and TGF- $\beta 1$ are two of the most-powerful and widely investigated molecules in various types of solid tumors, including pancreatic cancer ${ }^{31,36,37}$. Our data also indicated CAFs highly secreted SDF-1 compared with NAFs, which specifically binds to CXCR4 in pancreatic cancer cells and subsequently upregulates the expression of SATB-1 in pancreatic cancer cells.

SATB-1 is a nuclear matrix attachment region-binding protein located on chromosome $3 \mathrm{p} 23$, which regulates the global gene transcription and expression ${ }^{38}$. Since 2008, many researches gradually revealed that SATB-1 plays a crucial role in various types of malignant cancers, including breast cancer, laryngeal squamous cell 

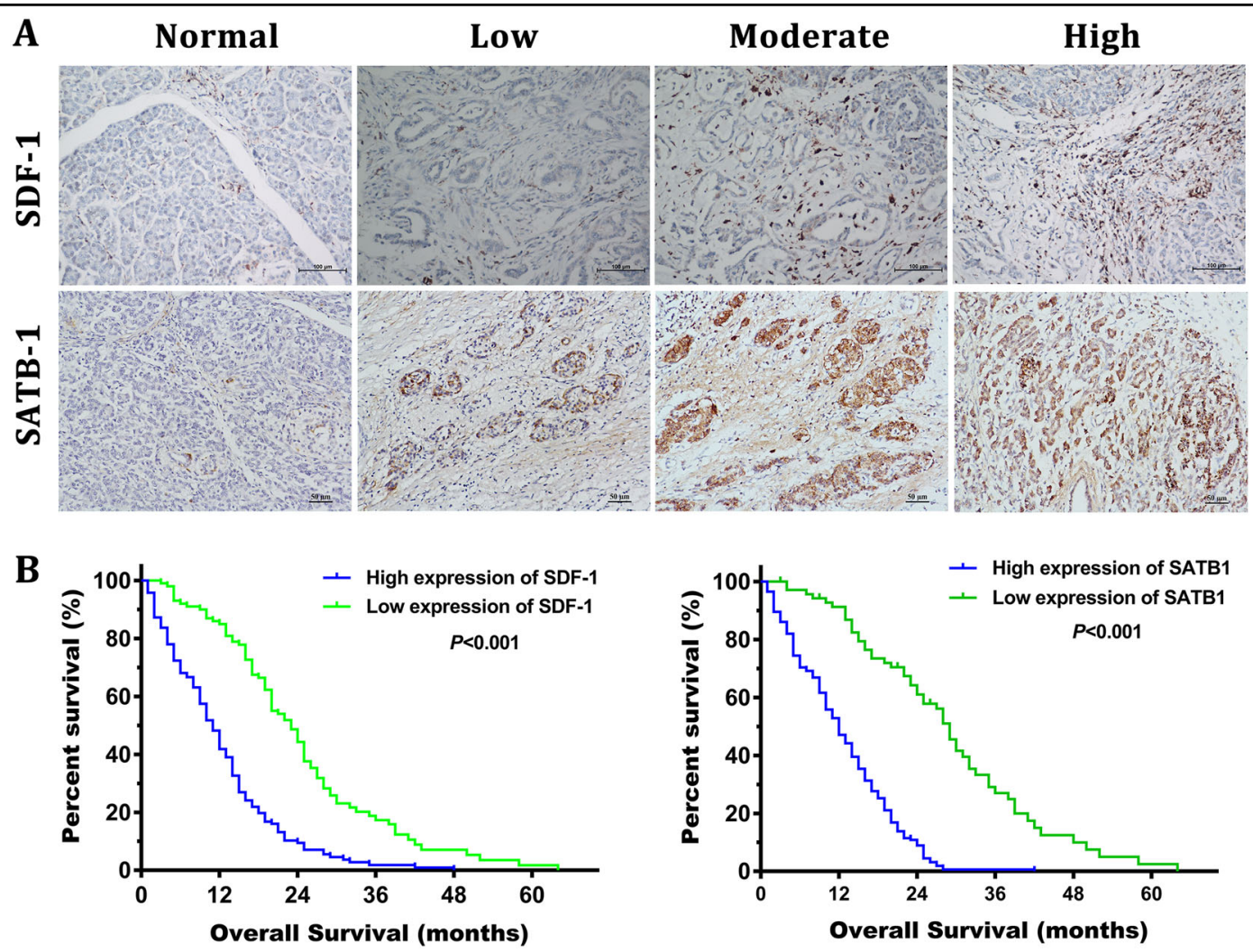

Fig. 9 Overexpression of SDF-1 and SATB-1 is correlated with clinicopathological characteristics and poor prognosis of PDAC patients a Representative images of SDF-1 and SATB-1 staining in PDAC tissue (intensity: low, moderate, and high). The expression of SDF-1 and SATB-1 were evaluated semi-quantitatively based on staining intensity and distribution according to immunoreactive score. $\mathbf{b}$ The Kaplan-Meier analysis of overall survival of PDAC patients stratified by the SDF-1 or SATB-1 immunoreactive scores. The log-rank test was performed to compare differences between groups

carcinoma $^{39}$, hepatocellular carcinoma $^{18}$, colorectal cancer $^{40,41}$, and gastric cancer ${ }^{42}$. However, the clinical and biological function of SATB-1 in pancreatic cancer deserves further investigation, especially in the tumor environment. As reported in our study, we found that SATB-1 is overexpressed in PDAC tissues and pancreatic cancer cell lines, and SATB-1 is upregulated by CAFs-secreted SDF-1. SATB-1 upregulation is relative with poor prognosis, which is similar to the inclusion of Chen Z's research ${ }^{27}$. Our clinical specimens IHC staining found that SATB-1 expression is relative with SDF-1 expression, suggesting SATB-1 expression in pancreatic cancer cells may be upregulated by SDF-1 derived from CAFs in the stromal. Besides, experimental models were utilized to validate the value of SATB-1 in pancreatic cancer, which mainly included the proliferation, migration, and invasion of pancreatic cancer cells in vitro. Further research, however, is needed to explore the molecular mechanism of SATB-1 effect on cancer cell behavior.

At early stages of tumorigenesis, carcinoma in situ already involves "reactive" tumor fibrosis ${ }^{7}$. The recruitment of fibroblasts to tumor is largely governed by growth factors secreted by cancer cells and infiltrating immune cells, including TGF- $\beta$, PDGF and fibroblast growth factor $2^{43}$. Although the function of CAFs is debated, they are generally viewed as contributors to the process of cancer initiation and progression. How cancer cells maintain the properties of CAFs in the reciprocal loop between cancer cells and CAFs is unclear. In our study, we found that SATB-1 expression in pancreatic cancer cells mediated the maintenance of CAF properties and the activation of CAFs from NAFs. However, the paracrine-secreted factors and a precise molecular mechanism are currently not fully understood and deserve further investigation.

Gemcitabine resistance is a persistent clinical challenge for pancreatic cancer therapy. CAFs acted as a key player in promoting cancer cell evasion of chemotherapy drugs $^{44}$. In this study, we revealed that the expression of SATB-1 mediated the gemcitabine sensibility of pancreatic cancer cells. SDF-1 has been revealed to take part in the development of chemotherapy resistance in various tumors, including breast cancer ${ }^{45}$, colorectal cancer ${ }^{46}$, glioblastoma ${ }^{47}$, acute myeloid leukemia ${ }^{48}$, and pancreatic cancer ${ }^{49}$. Feig et al. ${ }^{50}$ found that SDF-1 released by CAFs 
Table 2 Correlation between SATB-1 and SDF-1 expression and clinicopathological characterics of PDAC patients

\begin{tabular}{|c|c|c|c|c|c|c|c|}
\hline \multirow[t]{2}{*}{ Characteristics } & \multirow[t]{2}{*}{$\mathrm{N}$ of cases } & \multicolumn{3}{|c|}{ SATB-1 level } & \multicolumn{3}{|c|}{ SDF-1 level } \\
\hline & & $\mathbf{H}$ & $\mathbf{L}$ & $P$ value & $\mathbf{H}$ & $\mathrm{L}$ & $P$ value $^{\mathrm{a}}$ \\
\hline Total cases & 243 & 172 & 71 & & 141 & 102 & \\
\hline \multicolumn{8}{|l|}{ Sex } \\
\hline Male & 142 & 101 & 41 & 0.889 & 80 & 62 & 0.528 \\
\hline Female & 101 & 71 & 30 & & 61 & 40 & \\
\hline \multicolumn{8}{|l|}{ Age } \\
\hline$<60$ & 104 & 76 & 28 & 0.496 & 61 & 43 & 0.864 \\
\hline$\geq 60$ & 139 & 96 & 43 & & 80 & 59 & \\
\hline \multicolumn{8}{|l|}{ Differentiation } \\
\hline Poor & 68 & 58 & 10 & $0.008^{* *}$ & 46 & 22 & 0.158 \\
\hline Moderate & 118 & 78 & 40 & & 65 & 53 & \\
\hline Well & 57 & 36 & 21 & & 30 & 27 & \\
\hline \multicolumn{8}{|l|}{ T stage } \\
\hline $\mathrm{T} 1$ & 4 & 2 & 2 & $0.003^{* *}$ & 2 & 2 & 0.303 \\
\hline $\mathrm{T} 2$ & 28 & 15 & 13 & & 14 & 14 & \\
\hline T3 & 176 & 122 & 54 & & 100 & 76 & \\
\hline T4 & 35 & 32 & 3 & & 25 & 10 & \\
\hline \multicolumn{8}{|c|}{ TNM stage $(\mathrm{AJCC})^{\mathrm{b}}$} \\
\hline 1 & 17 & 10 & 7 & $0.025^{*}$ & 10 & 9 & 0.057 \\
\hline$\|$ & 130 & 88 & 57 & & 72 & 58 & \\
\hline III & 62 & 46 & 14 & & 32 & 28 & \\
\hline IV & 34 & 28 & 6 & & 27 & 7 & \\
\hline \multicolumn{8}{|c|}{ Lymph node metastasis } \\
\hline Positive & 147 & 119 & 28 & $0.000^{* *}$ & 99 & 48 & $0.000^{* *}$ \\
\hline Negative & 96 & 53 & 43 & & 42 & 54 & \\
\hline
\end{tabular}

${ }^{a}$ Chi-square test, ${ }^{*} P<0.05,{ }^{* *} P<0.01$

${ }^{b}$ American Joint Committee on Cancer (AJCC), patients were staged in accordance with the 7th Edition of the AJCC Cancer's' TNM Classification; $N$ of cases number of cases, $T$ stage tumor stage; TNM tumor node metastasis, $H$ high, L low

affected the anti-PD-1 immunotherapy. Our study emerged that CAF-promoted SATB-1 dysregulation in pancreatic cancer cells may contribute to gemcitabine resistance by some mechanism that requires further research.

In conclusion, we demonstrated that SDF-1-positive CAFs were significant in malignant progression and gemcitabine resistance, partially owing to paracrine induction of SATB-1 in pancreatic cancer cells. Overexpression of SATB-1 in pancreatic cancer cells was vital for the maintenance of CAF-like properties, thus forming a regulatory feedback loop in the tumor microenvironment. Our study provides the first evidence of the association of the SDF-1/CXCR4/SATB-1 axis with PDAC malignant progression and CAF maintenance, suggesting a potential new target of clinical interventions for pancreatic cancer patients.

\section{Materials and methods}

\section{Patients and clinical samples}

The 243 PDAC tissues were obtained from patients who underwent resection at Sun Yat-sen Memorial Hospital between 2006 and 2016. None of these patients received any preoperative chemotherapy or radiotherapy. The protocol was approved by the hospital's Protection of Human Subjects Committee, and written informed consent was obtained from all patients before surgery. All samples were immediately snap-frozen in liquid nitrogen and stored at $-80{ }^{\circ} \mathrm{C}$ until further use. All samples were histologically diagnosed with PDAC by pathologic examination. The detailed clinicopathologic characteristics of patients are summarized in Table 1. The collection of follow-up data was carried out completely, and overall survival was defined as the time interval from the date of surgery to the date of death or the end-point of follow-up (October 2017).

\section{Isolation of pancreatic CAFs and paired NAFs}

The isolation of stromal fibroblasts was previously described $^{11}$. In brief, surgically resected pancreatic cancer tissues were obtained from four patients with pancreatic ductal adenocarcinoma. The written informed consents were obtained before operation. The fresh pancreatic tumor tissue and adjacent normal tissue (at least $2 \mathrm{~cm}$ from the outer tumor margin) were minced into $1-3 \mathrm{~mm}^{3}$ fragments and digested with $1 \mathrm{mg} / \mathrm{ml}$ collagenase I (\#C0130, Sigma) at $37^{\circ} \mathrm{C}$ for $2 \mathrm{~h}$. The solution was centrifuged at $1000 \mathrm{rpm}$ for $5 \mathrm{~min}$, washed with phosphatebuffered saline twice and filtered with a $100-\mu \mathrm{m}$ filter. Then, the isolated cells were seeded in a $10-\mathrm{cm}$ dish. The details of the procedure can be found in the Supplementary Methods.

\section{Cell culture}

Human pancreatic cancer cell lines (CFPAC-1, HPAC-1, PANC-1, Capan-2, MIAPaCa-2, SW1990, and $\mathrm{BxPC}-3)$ were purchased from the American Type Culture Collection (ATCC, Manassas, USA). The details of the procedure can be found in the Supplementary Methods.

\section{RNA extraction and qRT-PCR}

Total RNA was extracted from frozen pancreatic cancer tissues and corresponding non-neoplastic tissues or cultured cell lines using TRIzol reagent (Invitrogen, Carlsbad, USA) according to the manufacturer's instructions. 
Table 3 Univariate and multivariate analysis of overall survival in PDAC patients $(n=243)$

\begin{tabular}{|c|c|c|c|c|c|c|c|}
\hline \multirow[t]{2}{*}{ Variables } & \multirow[t]{2}{*}{ Characteristics } & \multicolumn{3}{|c|}{ Univariate analysis } & \multicolumn{3}{|c|}{ Multivariate analysis } \\
\hline & & $H R$ & $95 \% \mathrm{Cl}$ & $P$ value & HR & $95 \% \mathrm{Cl}$ & $P$ value \\
\hline \multirow[t]{2}{*}{ Sex } & Male (ref) & 1.080 & $0.825-1.412$ & 0.576 & & & \\
\hline & Female & & & & & & \\
\hline \multirow[t]{2}{*}{ Age } & $<60$ (ref) & 1.000 & $0.767-1.304$ & 0.998 & & & \\
\hline & $\geq 60$ & & & & & & \\
\hline \multirow[t]{3}{*}{ Differentiation } & Poor (ref) & & & $0.009^{* *}$ & & & 0.292 \\
\hline & Moderate & 1.667 & $1.160-0.396$ & 0.006 & 1.344 & $0.914-1.976$ & 0.133 \\
\hline & Well & 1.105 & $0.794-1.537$ & 0.553 & 1.096 & $0.781-1.538$ & 0.595 \\
\hline \multirow[t]{4}{*}{ T stage } & T1 (ref) & & & $0.000^{* * *}$ & & & $0.005^{* *}$ \\
\hline & $\mathrm{T} 2$ & 0.232 & $0.081-0.665$ & 0.007 & 0.342 & $0.086-1.361$ & 0.128 \\
\hline & T3 & 0.186 & $0.108-0.323$ & 0.000 & 0.531 & $0.270-1.044$ & 0.067 \\
\hline & T4 & 0.275 & $0.186-0.408$ & 0.000 & 0.430 & $0.268-0.689$ & 0.000 \\
\hline \multirow[t]{4}{*}{ TNM stage } & I (ref) & & & $0.000^{* * *}$ & & & $0.000^{* * *}$ \\
\hline & $\|$ & 0.175 & $0.095-0.321$ & 0.000 & 0.154 & $0.067-0.357$ & 0.000 \\
\hline & III & 0.265 & $0.178-0.395$ & 0.000 & 0.348 & $0.219-0.552$ & 0.000 \\
\hline & IV & 0.461 & $0.299-0.710$ & 0.000 & 0.440 & $0.279-0.695$ & 0.000 \\
\hline \multirow[t]{2}{*}{ Lymph node metastasis } & Positive (ref) & 3.190 & $2.362-4.307$ & $0.000^{* * *}$ & 2.151 & $1.553-2.980$ & $0.000^{* * *}$ \\
\hline & Negative & & & & & & \\
\hline \multirow[t]{2}{*}{ SATB-1 expression } & High (ref) & 4.866 & 3.399-6.967 & $0.000^{* * *}$ & 3.473 & $2.305-5.235$ & $0.000^{* * *}$ \\
\hline & Low & & & & & & \\
\hline \multirow[t]{2}{*}{ SDF-1 expression } & High (ref) & 3.429 & $2.564-4.585$ & $0.000^{* * *}$ & 2.967 & $2.169-4.060$ & $0.000^{* * *}$ \\
\hline & Low & & & & & & \\
\hline
\end{tabular}

HR hazard ratio, 95\% Cl confidence interval, TNM tumor node metastasis, $T$ stage tumor stage, ref reference Cox regression analysis, Method: Enter, ${ }^{*} p<0.05,{ }^{* *} p<0.01,{ }^{* * *} p<0.001$

Table 4 The relationship between SDF-1 expression and SATB-1 expression in pancreatic cancer tissues

\begin{tabular}{lllll}
\hline & & \multicolumn{2}{l}{$\begin{array}{l}\text { SATB-1 } \\
\text { expression }\end{array}$} & Total \\
\cline { 3 - 4 } & & Low & High & \\
\hline SDF-1 expression & Low & 49 & 53 & 102 \\
& High & 22 & 119 & 141 \\
Total & & 71 & 172 & 243 \\
\hline
\end{tabular}

Pearson $x^{2}, p<0.001$

The procedural steps were performed as described in the Supplementary Methods. All primer sequences for qRTPCR are listed in Table S1 (see Additional file 1). For relative gene expression in tissues or cells, the levels were firstly normalized to $\beta$-actin expression as $\Delta \mathrm{Ct}$ and then compared with one of the tissues and converted to the fold change $\left(2^{-\Delta \Delta C t}\right)$.

\section{Cell transfection and viral infection}

For transient knockdown experiments, the siRNAs, CXCR4 siRNA (si-CXCR4), and synthetic sequencescrambled siRNA (si-NC), were purchased from GenePharma Co. (Shanghai, China). The stable suppression of SATB-1 was performed by short hairpin RNA interference. All oligonucleotide sequences are provided in Table S2 (see Additional file 2). The transfection and infection procedures are described in the Supplementary Methods.

\section{Expression construct}

The sequence of SATB-1 was synthesized and subcloned into pcDNA3.1 (Invitrogen, Shanghai, China). Ectopic expression of SATB-1 was achieved by using the pcDNA3.1SATB-1 transfection, and an empty pcDNA vector (pcDNA3.1-NC) was used as the control. The expression level of SATB-1 was detected by western blot analysis.

\section{Half-maximal IC50 assay}

Cells were collected and seeded in 96-well plates. The next day, fresh gemcitabine was added into cells at various 
concentrations of $0,0.01,0.1,0.5,1,5,10,40$, and $160 \mu \mathrm{M}$. After 72-h treatment, cells were photographed and quantified via CCK-8 assay. The half-maximal IC50 was calculated with the following formula: $\operatorname{Ig}(\mathrm{IC} 50)=\mathrm{Ig}$ $(\mathrm{Cmax})-\operatorname{Ig}(\mathrm{Cmax} / \mathrm{Csecond} \max ) \times(P-(3-P \max -P \min ) /$ 4), $P$ was for the sum of all positive rates, $P$ max was the maximal positive rate and $P$ min was the minimum positive rate.

\section{Western blot analysis}

Western blot assay was performed as described in the Supplementary Methods. Primary antibodies were: rabbit anti-human SATB-1 (1:1000, ab92307, Abcam), rabbit anti-human FAP (1:2000, ab28244, Abcam), rabbit antihuman $\alpha$-SMA (1:1000, ab32575, Abcam), and rabbit anti-human GAPDH antibody (1:2500, CW0101, Cwbio, China). GAPDH was used as a loading control. Horseradish peroxidase-linked secondary antibody was goat anti-rabbit IgG (1:5000; Cwbio, China).

\section{ELISA}

CAFs and matched NAFs $\left(10^{5}\right.$ cells/well $)$ were seeded in six-well plates and cultured in Dulbecco's Modified Eagle's medium without serum for 3 days. Conditioned mediums were collected and detected for SDF-1 and TGF- $\beta 1$ using ELISA kits (Cat: DSA00 and DB100B, R\&D, USA) according to the manufacturer's instructions. Each experiment was repeated at least three times.

\section{Immunohistochemical analysis}

Paraffin-embedded samples of primary carcinomas were immunostained with primary rabbit anti-human SATB-1 (1:100, ab92307, Abcam), rabbit anti-human $\alpha$-SMA (1:100, ab32575, Abcam), and rabbit anti-human SDF-1 (1:500, ab9797, Abcam) and Ki-67 (1:500, ab6526, Abcam) antibodies. The expression levels of SATB-1 and SDF-1 were scored semi-quantitatively, based on staining intensity and distribution, using the immunoreactive score as described elsewhere. Staining was assessed by two pathologists under double-blind conditions according to the scoring criteria. These procedures are described in the Supplementary Methods.

\section{Tumor formation assay}

The athymic BALB/c nude mice (4-6 weeks old) were used for the tumor formation assay. SW1990 cells $(2 \times$ $10^{6}$ ) were injected subcutaneously into left and right bilateral hind legs of mice. Tumor volume was calculated as follows: Volume $=\left(L \times W^{2}\right) / 2(V$, volume; $L$, length diameter; $W$, width diameter). The animal care and experimental protocols were approved by the institutional guidelines of Guangzhou Province and by the Use Committee for Animal Care. All necessary steps were taken to minimize the suffering and distress caused to the mice.
The procedures of tumor formation assay are described in the Supplementary Methods.

\section{Statistical analysis}

All statistical analyses were performed using SPSS Statistics 18.0 software (IBM Chicago, IL, USA). The chisquare test $\left(\chi^{2}\right.$-test) for nonparametric variables, and the two-tailed Student's $t$ test or one-way analysis of variance for parametric variables were used. All data are presented as the mean $\pm \mathrm{SD}$ from at least three independent experiments, unless otherwise noted. Differences in patient survival were assessed using the Kaplan-Meier method and analyzed using the log-rank test in a univariate analysis. Univariate and multivariate Cox regression analyses were performed to assess the relative risk for each factor. All tests were two-sided, and results with $p<$ 0.05 were considered statistically significant.

\section{Acknowledgements}

This study was supported by grants from the National Natural Science Foundation of China (grant number 81672395, 81702951, 81672395, 81672807, 81702417, 81000917, and 81402213, 81370059 www.nsfc.gov.cn), the Guangdong Science and Technology Department (grant number S2012010008934, 2014A030313044, 2014A030311047, 2016A030313340, and 2016A030313296, 2017A030313880, http://pro.gdstc.gov.cn), the Sun Yat-sen University Clinical Research Foundation of 5010 Project (grant number 2012007). Grant from Guangdong Science and Technology Department (2015B050501004); Grant [2013] 163 from Key Laboratory of Malignant Tumor Molecular Mechanism and Translational Medicine of Guangzhou Bureau of Science and Information Technology; Grant KLB09001 from the Key Laboratory of Malignant Tumor Gene Regulation and Target Therapy of Guangdong Higher Education Institutes.

\section{Author details}

${ }^{1}$ Guangdong Provincial Key Laboratory of Malignant Tumor Epigenetics and Gene Regulation, Sun Yat-sen Memorial Hospital, Sun Yat-sen University, Guangzhou, Guangdong Province, China. ${ }^{2}$ Department of Pancreatobiliary Surgery, Sun Yat-sen Memorial Hospital, Sun Yat-sen University, Guangzhou, Guangdong Province, China. ${ }^{3}$ Department of Radiology, Guangzhou women and children's medical center, Guangzhou Medical University, Guangzhou, Guangdong Province, China. ${ }^{4}$ Department of Radiotherapy, Sun Yat-sen Memorial Hospital, Sun Yat-sen University, Guangzhou, Guangdong Province, China. ${ }^{5}$ Department of Medical Oncology, Sun Yat-sen Memorial Hospital, Sun Yat-sen University, Guangzhou, Guangdong Province, China

\section{Conflict of interest}

The authors declare no conflict of interest.

\section{Ethics approval and consent to participate}

The studies involved with human PDAC tissue samples were approved by ethics committee of Sun Yat-sen Memorial Hospital, Sun Yat-sen University. Informed consent from the patients was obtained in all cases. The protocols for animal experiment were approved by the Institutional Animal Care and Use Committee and Institutional Biosafety Committee of Sun Yat-sen University.

\section{Publisher's note}

Springer Nature remains neutral with regard to jurisdictional claims in published maps and institutional affiliations.

Supplementary Information accompanies this paper at (https://doi.org/ 10.1038/s41419-018-1104-x). 
Received: 1 April 2018 Revised: 26 September 2018 Accepted: 26 September 2018

Published online: 18 October 2018

\section{References}

1. Siegel, R. L., Miller, K. D. \& Jemal, A. Cancer Statistics, 2017. CA Cancer J. Clin. 67 7-30 (2017)

2. Rahib, L. et al. Projecting cancer incidence and deaths to 2030: the unexpected burden of thyroid, liver, and pancreas cancers in the United States. Cancer Res. 74, 2913-2921 (2014).

3. Wolfgang, C. L. et al. Recent progress in pancreatic cancer. CA Cancer J. Clin. 63, 318-348 (2013).

4. Oettle, H. et al. Adjuvant chemotherapy with gemcitabine vs observation in patients undergoing curative-intent resection of pancreatic cancer: a randomized controlled trial. JAMA 297, 267-277 (2007).

5. Erkan, $\mathbf{M}$. et al. The activated stroma index is a novel and independent prognostic marker in pancreatic ductal adenocarcinoma. Clin. Gastroenterol. Hepatol. 6, 1155-1161 (2008).

6. Ronnov-Jessen, L., Petersen, O. W. \& Bissell, M. J. Cellular changes involved in conversion of normal to malignant breast: importance of the stromal reaction. Physiol. Rev. 76, 69-125 (1996).

7. Kalluri, R. The biology and function of fibroblasts in cancer. Nat. Rev. Cancer 16, 582 (2016).

8. von Ahrens, D., Bhagat, T. D., Nagrath, D., Maitra, A. \& Verma, A. The role of stromal cancer-associated fibroblasts in pancreatic cancer. J. Hematol. Oncol. 10, 76 (2017)

9. Cirri, P. \& Chiarugi, P. Cancer-associated-fibroblasts and tumour cells: a diabolic liaison driving cancer progression. Cancer Metastasis Rev. 31, 195-208 (2012).

10. Nair, N. et al. A cancer stem cell model as the point of origin of cancerassociated fibroblasts in tumor microenvironment. Sci. Rep. 7, 6838 (2017).

11. Richards, K. E. et al. Cancer-associated fibroblast exosomes regulate survival and proliferation of pancreatic cancer cells. Oncogene 36, 1770-1778 (2017).

12. De, W. O., Van, B. M., Mareel, M., Hendrix, A. \& Bracke, M. Carcinoma-associated fibroblasts provide operational flexibility in metastasis. Semin Cancer Biol. 25 33 (2014)

13. Yasui, D., Miyano, M., Cai, S., Varga-Weisz, P. \& Kohwi-Shigematsu, T. SATB-1 targets chromatin remodelling to regulate genes over long distances. Nature 419, 641-645 (2002).

14. Cai, S., Han, H. J. \& Kohwi-Shigematsu, T. Tissue-specific nuclear architecture and gene expression regulated by SATB-1. Nat. Genet. 34, 42-51 (2003).

15. Burute, M., Gottimukkala, K. \& Galande, S. Chromatin organizer SATB-1 is an important determinant of T-cell differentiation. Immunol. Cell Biol. 90, 852-859 (2012).

16. Savarese, F. et al. Satb1 and Satb2 regulate embryonic stem cell differentiation and Nanog expression. Genes Dev. 23, 2625-2638 (2009).

17. Han, H. J., Russo, J., Kohwi, Y. \& Kohwi-Shigematsu, T. SATB-1 reprogrammes gene expression to promote breast tumour growth and metastasis. Nature 452, 187-193 (2008).

18. Tu, W. et al. Upregulation of SATB-1 promotes tumor growth and metastasis in liver cancer. Liver Int. 32, 1064-1078 (2012).

19. Hedner, C. et al. SATB-1 is an independent prognostic factor in radically resected upper gastrointestinal tract adenocarcinoma. Virchows Arch. 465 , 649-659 (2014).

20. Cheng, C. et al. Overexpression of SATB-1 is associated with biologic behavior in human renal cell carcinoma. PLOS ONE 9, e97406 (2014).

21. Wan, F. et al. SATB1 overexpression regulates the development and progression in bladder cancer through EMT. PLOS ONE 10, e117518 (2015).

22. Mao, L. J. et al. SATB1 promotes prostate cancer metastasis by the regulation of epithelial-mesenchymal transition. Biomed. Pharmacother. 79, 1-8 (2016).

23. Mir, R., Pradhan, S. J., Patil, P., Mulherkar, R. \& Galande, S. Wnt/beta-catenin signaling regulated SATB1 promotes colorectal cancer tumorigenesis and progression. Oncogene 35, 1679-1691 (2016).

24. Li, Y. C. et al. SATB1 promotes tumor metastasis and invasiveness in oral squamous cell carcinoma. Oral Dis. 23, 247-254 (2017).
25. Nodin, B., Hedner, C., Uhlen, M. \& Jirstrom, K. Expression of the global regulator SATB1 is an independent factor of poor prognosis in high grade epithelial ovarian cancer. J. Ovarian Res. 5, 24 (2012).

26. Guo, L., Zheng, J., Yu, T., Liu, Y. \& Duo, L. Elevated expression of SATB1 is involved in pancreatic tumorigenesis and is associated with poor patient survival. Mol. Med. Rep. 16, 8842-8848 (2017).

27. Chen, Z. et al. SATB1 promotes pancreatic cancer growth and invasion depending on MYC activation. Dig. Dis. Sci. 60, 3304-3317 (2015).

28. Fuyuhiro, Y. et al. Upregulation of cancer-associated myofibroblasts by TGFbeta from scirrhous gastric carcinoma cells. Br. J. Cancer 105, 996-1001 (2011).

29. Mir, R., Pradhan, S. J. \& Galande, S. Chromatin organizer SATB1 as a novel molecular target for cancer therapy. Curr. Drug Targets 13, 1603-1615 (2012).

30. Kohwi-Shigematsu, T. et al. Genome organizing function of SATB1 in tumor progression. Semin Cancer Biol. 23, $72-79$ (2013).

31. Janssens, R., Struyf, S. \& Proost, P. The unique structural and functional features of CXCL12. Cell Mol. Immunol. 15, 299-311 (2018).

32. Zheng, X. et al. Epithelial-to-mesenchymal transition is dispensable for metastasis but induces chemoresistance in pancreatic cancer. Nature 527, 525-530 (2015).

33. Qin, X. et al. Cancer-associated fibroblast-derived IL-6 promotes head and neck cancer pogression via the osteopontin-NF-kappa B signaling pathway. Theranostics 8, 921-940 (2018).

34. Ren, Y. et al. Paracrine and epigenetic control of CAF-induced metastasis: the role of HOTAIR stimulated by TGF-ss1 secretion. Mol. Cancer 17, 5 (2018).

35. Alba-Castellon, L. et al. Snail1-dependent activation of cancer-associated fibroblast controls epithelial tumor cell invasion and metastasis. Cancer Res. 76, 6205-6217 (2016).

36. Sleightholm, R. L. et al. Emerging roles of the CXCL12/CXCR4 axis in pancreatic cancer progression and therapy. Pharmacol. Ther. 179, 158-170 (2017).

37. Seoane, J. \& Gomis, R. R. TGF-beta family signaling in tumor suppression and cancer progression. Cold Spring Harb. Perspect. Biol. 9, pii: a022277 (2017).

38. Dickinson, L. A., Joh, T., Kohwi, Y. \& Kohwi-Shigematsu, T. A tissue-specific MAR/SAR DNA-binding protein with unusual binding site recognition. Cell 70, 631-645 (1992).

39. Selinger, C. I. et al. Loss of special AT-rich binding protein 1 expression is a marker of poor survival in lung cancer. J. Thorac. Oncol. 6, 1179-1189 (2011).

40. Zhang, Y. et al. Expression of SATB1 promotes the growth and metastasis of colorectal cancer. PLOS ONE 9, e100413 (2014).

41. Al-Sohaily, S. et al. Loss of special AT-rich sequence-binding protein 1 (SATB1) predicts poor survival in patients with colorectal cancer. Histopathology 65 155-163 (2014).

42. Cheng, C. et al. Expression of SATB1 and heparanase in gastric cancer and its relationship to clinicopathologic features. APMIS 118, 855-863 (2010).

43. Elenbaas, B. \& Weinberg, R. A. Heterotypic signaling between epithelial tumor cells and fibroblasts in carcinoma formation. Exp. Cell Res. 264, 169-184 (2001).

44. Paraiso, K. H. \& Smalley, K. S. Fibroblast-mediated drug resistance in cancer. Biochem. Pharmacol. 85, 1033-1041 (2013).

45. Ham, S. L. et al. Three-dimensional tumor model mimics stromal - breast cancer cells signaling. Oncotarget 9, 249-267 (2018).

46. Jung, $K$. et al. Targeting CXCR4-dependent immunosuppressive Ly6C(low) monocytes improves antiangiogenic therapy in colorectal cancer. Proc. Natl. Acad. Sci. USA 114, 10455-10460 (2017).

47. Calinescu, A. A. et al. Survival and proliferation of neural progenitor-derived glioblastomas under hypoxic stress is controlled by a CXCL12/CXCR4 autocrine-positive feedback mechanism. Clin. Cancer Res. 23, 1250-1262 (2017).

48. Cho, B. S. et al. Antileukemia activity of the novel peptidic CXCR4 antagonist LY2510924 as monotherapy and in combination with chemotherapy. Blood 126, 222-232 (2015).

49. Morimoto, M. et al. Enhancement of the CXCL12/CXCR4 axis due to acquisition of gemcitabine resistance in pancreatic cancer: effect of CXCR4 antagonists. BMC Cancer 16, 305 (2016).

50. Feig, C. et al. Targeting CXCL12 from FAP-expressing carcinoma-associated fibroblasts synergizes with anti-PD-L1 immunotherapy in pancreatic cancer. Proc. Natl. Acad. Sci. USA 110, 20212-20217 (2013). 